\title{
Exponentially Fitted and Trigonometrically Fitted Explicit Modified Runge-Kutta Type Methods for Solving $y^{\prime \prime \prime}(x)=f\left(x, y, y^{\prime}\right)$
}

\author{
N. Ghawadri, ${ }^{1}$ N. Senu $\mathbb{D},{ }^{1,2}$ F. Ismail $\mathbb{D},{ }^{1,2}$ and Z. B. Ibrahim $\mathbb{D}^{1,2}$ \\ ${ }^{1}$ Institute for Mathematical Research, Universiti Putra Malaysia (UPM), 43400 Serdang, Selangor, Malaysia \\ ${ }^{2}$ Department of Mathematics, Universiti Putra Malaysia (UPM), 43400 Serdang, Selangor, Malaysia \\ Correspondence should be addressed to N. Senu; norazak@upm.edu.my
}

Received 21 December 2017; Revised 1 May 2018; Accepted 28 May 2018; Published 4 July 2018

Academic Editor: Igor Andrianov

Copyright ( $\odot 2018$ N. Ghawadri et al. This is an open access article distributed under the Creative Commons Attribution License, which permits unrestricted use, distribution, and reproduction in any medium, provided the original work is properly cited.

Exponentially fitted and trigonometrically fitted explicit modified Runge-Kutta type (MRKT) methods for solving $y^{\prime \prime \prime}(x)=$ $f\left(x, y, y^{\prime}\right)$ are derived in this paper. These methods are constructed which exactly integrate initial value problems whose solutions are linear combinations of the set functions $e^{\omega x}$ and $e^{-\omega x}$ for exponentially fitted and $\sin (\omega x)$ and $\cos (\omega x)$ for trigonometrically fitted with $\omega \in R$ being the principal frequency of the problem and the frequency will be used to raise the accuracy of the methods. The new four-stage fifth-order exponentially fitted and trigonometrically fitted explicit MRKT methods are called EFMRKT5 and TFMRKT5, respectively, for solving initial value problems whose solutions involve exponential or trigonometric functions. The numerical results indicate that the new exponentially fitted and trigonometrically fitted explicit modified Runge-Kutta type methods are more efficient than existing methods in the literature.

\section{Introduction}

This work deals with exponentially fitted and trigonometrically fitted modified Runge-Kutta type methods for solving third-order ordinary differential equations (ODEs)

$$
\begin{aligned}
y^{\prime \prime \prime}(x) & =f\left(x, y(x), y^{\prime}(x)\right), \\
y\left(x_{0}\right) & =y_{0}, \\
y^{\prime}\left(x_{0}\right) & =y_{0}^{\prime} \\
y^{\prime \prime}\left(x_{0}\right) & =y_{0}^{\prime \prime}
\end{aligned}
$$

$$
x \geq x_{0} .
$$

This sort of problems is often found in numerous physical problems like thin film flow, gravity-driven flows, electromagnetic waves, and so on. In the past and recent years many researchers constructed exponentially fitted and trigonometrically fitted explicit Runge-Kutta methods for solving first-order and second-order ordinary differential equations.
Paternoster [1] developed Runge-Kutta-Nyström methods for ODEs with periodic solutions based on trigonometric polynomials. Vanden Berghe et al. [2] developed exponentially fitted Runge-Kutta methods. Simos [3] extended exponentially fitted Runge-Kutta methods for the numerical solution of the Schrodinger equation and related problems. Kalogiratou et al. $[[4,5]]$ constructed trigonometrically and exponentially fitted Runge-Kutta-Nyström methods for the numerical solution of the Schrodinger equation and related problems which is eighth algebraic order. Next Simos et al. [6] constructed exponentially fitted Runge-Kutta-Nyström method for the numerical solution of initial value problems with oscillating solutions. Sakas et al. [7] developed a fifth algebraic order trigonometrically fitted modified Runge-Kutta Zonneveld method for the numerical solution of orbital problems. Van de Vyver [8] in 2005 constructed Runge-Kutta-Nyström pair for the numerical integration of perturbed oscillators. Then Yang et al. [9] constructed trigonometrically fitted adapted Runge-Kutta-Nyström methods for perturbed oscillators. Recently, Demba et al. [10] constructed an explicit 
trigonometrically fitted Runge-Kutta-Nyström method using Simos technique.

In this paper we construct explicit exponentially fitted and trigonometrically fitted modified Runge-Kutta type methods with four-stage fifth-order, called EFMRKT5 and TFMRKT5, respectively. Section 2 discussed the oscillatory and nonoscillatory properties of the third-order linear differential equation. In Section 3, the necessary conditions and the derivation for exponentially fitted and trigonometrically fitted modified Runge-Kutta type methods for solving thirdorder ODEs are given. The error analysis of the new EFMRKT5 and TFMRKT5 methods was discussed in Section 4, respectively. The effectiveness of the new methods when compared with existing methods is given in Section 5. The thin film flow problem is discussed in Section 6.

\section{Third-Order Linear Differential Equation with Oscillating and Nonoscillating Solutions}

This section discusses the oscillatory and nonoscillatory properties of the third-order linear differential equation

$$
y^{\prime \prime \prime}(x)+p(x) y^{\prime}+q(x) y=0 .
$$

A solution of (2) will be said to be oscillatory if it changes signs for arbitrarily large values of $x$. The other solutions will be said to be nonoscillatory.

If $p(x)<0$ and $q(x)<0$ are constants, then it is easy to show that if (2) has an oscillatory solution, then there are two linearly independent oscillatory solutions of (2) whose zeroes separate and such that any oscillatory solution of (2) is a linear combination of them. Assuming that $p(x), p^{\prime}(x)$, and $q(x)$ are continuous on $[0,+\infty)$ the following will be established (see [11-14]).

Definition 1. A solution of (2) will be called oscillatory iff it has an infinity of zeroes in $(0,+\infty)$ and nonoscillatory iff it has but a finite number of zeroes in this interval. Equation (2) is said to be oscillatory iff it has at least one (nontrivial) oscillatory solution and nonoscillatory iff all of its (nontrivial) solutions are nonoscillatory.

Particularly, this paper deals with two cases based on (2) when $q(x)=0$, as follows:

(i) $y^{\prime \prime \prime}(x)=p y^{\prime},(p>0)$; it is clear that the characteristic roots equations are real and one of them is zero; then solutions will consist of exponential functions.

(ii) $y^{\prime \prime \prime}(x)=-p y^{\prime},(p>0)$; one of the characteristic roots equations is zero and another two are conjugate roots and the solutions are in oscillatory form, where $p$ is constant.

\section{Exponentially Fitted and Trigonometrically Fitted MRKT Methods}

In this section, we will determine the conditions and develop exponentially fitted and trigonometrically fitted MRKT
TABLE 1: The Butcher tableau MRKT method.

\begin{tabular}{llllll}
\hline$c$ & $\gamma$ & $\widehat{\gamma}$ & $A$ & $\widehat{A}$ & \\
\hline & & & $b$ & $b^{\prime}$ & $b^{\prime \prime}$ \\
\hline
\end{tabular}

methods. In order to construct the exponentially fitted and trigonometrically fitted MRKT methods, the extra $\gamma_{i}$ and $\widehat{\gamma}_{i}$ are absolutely necessary to insert at each stage and the MRKT methods is given as follows:

$$
\begin{aligned}
& y_{n+1}=y_{n}+h y_{n}^{\prime}+\frac{h^{2}}{2} y_{n}^{\prime \prime}+h^{3} \sum_{i=1}^{s} b_{i} k_{i}, \\
& y_{n+1}^{\prime}=y_{n}^{\prime}+h y_{n}^{\prime \prime}+h^{2} \sum_{i=1}^{s} b_{i}^{\prime} k_{i}, \\
& y_{n+1}^{\prime \prime}=y_{n}^{\prime \prime}+h \sum_{i=1}^{s} b_{i}^{\prime \prime} k_{i},
\end{aligned}
$$

where

$$
\begin{aligned}
k_{1} & =f\left(x_{n}, y_{n}, y_{n}^{\prime}\right), \\
k_{i} & =f\left(x_{n}+c_{i} h, \gamma_{i} y_{n}+h c_{i} y_{n}^{\prime}+\frac{h^{2}}{2} c_{i}^{2} y_{n}^{\prime \prime}\right. \\
& \left.+h^{3} \sum_{j=1}^{s} a_{i j} k_{j}, y_{n}^{\prime}+\widehat{\gamma}_{i} h c_{i} y_{n}^{\prime \prime}+h^{2} \sum_{j=1}^{s} \widehat{a}_{i j} k_{j}\right)
\end{aligned}
$$

for $i=2,3, \ldots, s$.

The parameters of the MRKT methods are $c_{i}, a_{i j}, \widehat{a}_{i j}, b_{i}$, $b_{i}^{\prime}, b_{i}^{\prime \prime}, \gamma_{i}$ and $\widehat{\gamma}_{i}$ for $i=1,2, \ldots, s$ and $j=1,2, \ldots, s$ are assumed to be real. If $a_{i j}=0$ and $\widehat{a}_{i j}=0$ for $i \leqslant j$, it is an explicit method and otherwise implicit method.

The MRKT method can be expressed in Butcher notation using the table of coefficients as follows (see Table 1).

3.1. Exponentially Fitted MRKT Method. To construct the exponentially fitted Runge-Kutta type four-stage fifth-order method the functions $e^{\omega x}$ and $e^{-\omega x}$ need to integrate exactly at each stage; therefore the following four equations are obtained:

$$
\begin{aligned}
& e^{ \pm c_{i} v}=\gamma_{i} \pm c_{i} v+\frac{1}{2} c_{i}^{2} v^{2} \pm v^{3} \sum_{j=1}^{s} a_{i j} e^{ \pm c_{j} v}, \\
& e^{ \pm c_{i} v}=1 \pm \widehat{\gamma}_{i} c_{i} v \pm v^{2} \sum_{j=1}^{s} \widehat{a}_{i j} e^{ \pm c_{j} v},
\end{aligned}
$$

and six more equations corresponding to $y, y^{\prime}$, and $y^{\prime \prime}$ :

$$
\begin{aligned}
& e^{ \pm v}=1 \pm v+\frac{1}{2} v^{2} \pm v^{3} \sum_{i=1}^{s} b_{i} e^{ \pm c_{i} v}, \\
& e^{ \pm v}=1 \pm v+v^{2} \sum_{i=1}^{s} b_{i}^{\prime} e^{ \pm c_{i} v},
\end{aligned}
$$




$$
e^{ \pm v}=1 \pm v \sum_{i=1}^{s} b_{i}^{\prime \prime} e^{ \pm c_{i} v}
$$

where $v=\omega h, \omega \in \mathrm{R}$. The relations $\cosh (v)=\left(e^{v}+e^{-v}\right) / 2$ and $\sinh (v)=\left(e^{v}-e^{-v}\right) / 2$ will be used in the derivation process. The following order conditions are obtained:

$$
\begin{aligned}
& \cosh \left(v c_{i}\right)=\gamma_{i}+\frac{1}{2} v^{2} c_{i}^{2}+v^{3} \sum_{j=1}^{i-1} a_{i j} \sinh \left(v c_{j}\right), \\
& \sinh \left(v c_{i}\right)=v c_{i}+v^{3} \sum_{j=1}^{i-1} a_{i j} \cosh \left(v c_{j}\right), \\
& \cosh \left(v c_{i}\right)=1+v^{2} \sum_{j=1}^{i-1} \widehat{a}_{i j} \cosh \left(v c_{j}\right), \\
& \sinh \left(v c_{i}\right)=\widehat{\gamma}_{i} c_{i} v+v^{2} \sum_{j=1}^{i-1} \widehat{a}_{i j} \sinh \left(v c_{j}\right),
\end{aligned}
$$

and six equations corresponding to $y, y^{\prime}$, and $y^{\prime \prime}$ :

$$
\begin{aligned}
& \cosh (v)=1+\frac{1}{2} v^{2}+v^{3} \sum_{i=1}^{s} b_{i} \sinh \left(v c_{i}\right) \\
& \sinh (v)=v+v^{3} \sum_{i=1}^{s} b_{i} \cosh \left(v c_{i}\right), \\
& \cosh (v)=1+v^{2} \sum_{i=1}^{s} b_{i}^{\prime} \cosh \left(v c_{i}\right) \\
& \sinh (v)=v+v^{2} \sum_{i=1}^{s} b_{i}^{\prime} \sinh \left(v c_{i}\right) \\
& \cosh (v)=1+v \sum_{i=1}^{s} b_{i}^{\prime \prime} \sinh \left(v c_{i}\right), \\
& \sinh (v)=v \sum_{i=1}^{s} b_{i}^{\prime \prime} \cosh \left(v c_{i}\right) .
\end{aligned}
$$

Solving (13) to (16), we find $a_{i, i-1}, \widehat{a}_{i, i-1}, \gamma_{i}$, and $\widehat{\gamma}_{i}$.

$$
\begin{array}{r}
\gamma_{i}=\cosh \left(v c_{i}\right)-\frac{1}{2} v^{2} c_{i}^{2}-v^{3} \sum_{j=1}^{i-1} a_{i, j} \sinh \left(v c_{j}\right), \\
a_{i, i-1}=\frac{\sinh \left(v c_{i}\right)-v c_{i}-v^{3} \sum_{j=1}^{i-2} a_{i, j} \cosh \left(v c_{j}\right)}{v^{3} \cosh \left(v c_{i-1}\right)}, \\
\widehat{a}_{i, i-1}=\frac{\cosh \left(v c_{i}\right)-1-v^{2} \sum_{j=1}^{i-2} \widehat{a}_{i, j} \cosh \left(v c_{j}\right)}{v^{2} \cosh \left(v c_{i-1}\right)}, \\
\widehat{a}_{21}=\frac{\cosh (v / 5)-1}{v^{2}}, \\
\widehat{a}_{32}=\frac{\cosh (2 v / 3)-1+(1 / 27) v^{2}}{v^{2} \cosh (v / 5)},
\end{array}
$$

$$
\widehat{\gamma}_{i}=\frac{\sinh \left(v c_{i}\right)-v^{2} \sum_{j=1}^{i-2} \widehat{a}_{i, j} \sinh \left(v c_{j}\right)}{v c_{i}}
$$

$$
i=2, \ldots, s \text {. }
$$

Referring to the following fifth-order four-stage method developed by Fawzi et al. [15]:

$$
\begin{aligned}
c_{1} & =0, \\
c_{2} & =\frac{1}{5}, \\
c_{3} & =\frac{2}{3}, \\
c_{4} & =1, \\
a_{21} & =0, \\
a_{31} & =-\frac{49}{4860}, \\
a_{41} & =\frac{7}{50}, \\
a_{42} & =-\frac{1}{50}, \\
\widehat{a}_{31} & =-\frac{1}{27}, \\
\widehat{a}_{41} & =\frac{3}{10}, \\
\widehat{a}_{42} & =-\frac{2}{35}, \\
b_{3} & =\frac{3}{112}, \\
b_{4} & =0, \\
b_{3}^{\prime} & =\frac{9}{56}, \\
b_{4}^{\prime} & =0, \\
b_{3}^{\prime \prime} & =\frac{7}{56}, \\
b_{4}^{\prime} & =\frac{5}{48},
\end{aligned}
$$

we solve (23) to (26) and let $\widehat{a}_{21}, \widehat{a}_{32}, \widehat{a}_{43}, a_{32}, a_{43}, \gamma_{2}, \gamma_{3}, \gamma_{4}$, $\widehat{\gamma}_{2}, \widehat{\gamma}_{3}$, and $\widehat{\gamma}_{4}$ be free parameters and yields. 


$$
\begin{aligned}
& \widehat{a}_{43}= \frac{\cosh (v)-1-v^{2}(3 / 10-(2 / 35) \cosh (v / 5))}{v^{2} \cosh (2 v / 3)}, \\
& a_{32}= \frac{\sinh (2 v / 3)-2 v / 3+(49 / 4860) v^{3}}{v^{3} \cosh \left(c_{2} v\right)}, \\
& a_{43}= \frac{\sinh (v)-v-(7 / 50) v^{3}+(1 / 50) v^{3} \cosh (v / 5)}{v^{3} \cosh (2 v / 3)}, \\
& \gamma_{2}= \cosh \left(\frac{v}{5}\right)-\frac{v^{2}}{50}, \\
& \gamma_{3}=\cosh \left(\frac{2 v}{3}\right)-\frac{2 v^{2}}{9}-\frac{301 v^{3}}{4860} \sinh \left(\frac{v}{5}\right), \\
& \gamma_{4}=\cosh (v)-\frac{v^{2}}{2}-v^{3}\left(-\frac{1}{50} \sinh \left(\frac{v}{5}\right)+\frac{1}{25} \sinh \left(\frac{2 v}{3}\right)\right), \\
& \widehat{\gamma}_{2}=\frac{5}{v} \sinh \left(\frac{v}{5}\right), \\
& \widehat{\gamma}_{3}=\frac{2}{3 v} \sinh \left(\frac{2 v}{3}\right)-\frac{2\left(\cosh (2 v / 3)-1+v^{2} / 27\right) \sinh (v / 5)}{3 \cosh (v / 5) v^{2}}, \\
& \\
& \frac{1}{35} \sinh \left(\frac{v}{5}\right)-\frac{\left.\cosh (v)-1-\left(3 v^{2} / 10-\left(2 v^{2} / 35\right) \cosh (v / 5)\right)\right) \sinh (2 v / 3)}{v \cosh (2 v / 3)} .
\end{aligned}
$$

Next, we solve (17) to (22) and use the above coefficients to find $b_{1}, b_{2}, b_{1}^{\prime}, b_{2}^{\prime}, b_{1}^{\prime \prime}$, and $b_{2}^{\prime \prime}$.

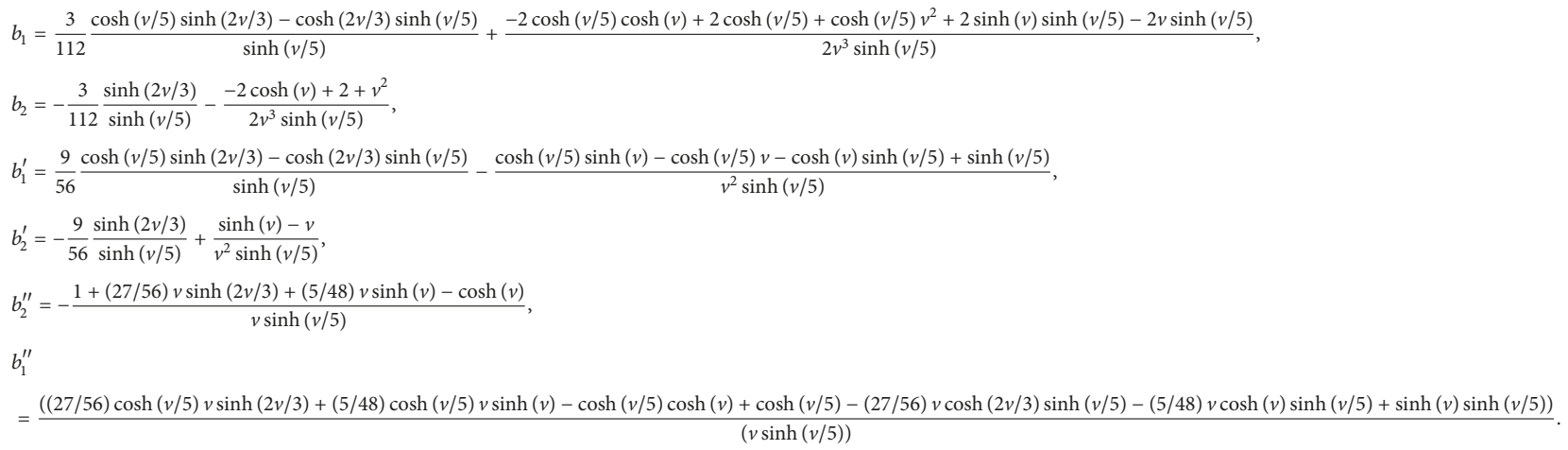

These lead to our new exponentially fitted Runge-Kutta type four-stage fifth-order explicit MRKT method denoted as EFMRKT5. The corresponding Taylor series expansion of the solution is given by

$$
\begin{aligned}
b_{1}= & \frac{1}{48}+\frac{1}{2160} v^{2}+\frac{101}{136080000} v^{4} \\
& -\frac{5713}{183708000000} v^{6} \\
& -\frac{11330339}{81841914000000000} v^{8}
\end{aligned}
$$

$$
-\frac{57722879}{134057055132000000000} v^{10}+\cdots,
$$$$
b_{2}=\frac{5}{42}-\frac{1}{2160} v^{2}-\frac{2921}{136080000} v^{4}
$$$$
-\frac{1361}{45927000000} v^{6}
$$$$
+\frac{54293587}{40920957000000000} v^{8}
$$$$
+\frac{6964030429}{670285275660000000000} v^{10}+\cdots,
$$ 


$$
\begin{aligned}
& b_{1}^{\prime}=\frac{1}{24}-\frac{17}{283500} v^{4}-\frac{149}{218700000} v^{6} \\
& -\frac{1055069}{341007975000000} v^{8} \\
& -\frac{23025689}{2538959377500000000} v^{10}+\cdots, \\
& b_{2}^{\prime}=\frac{25}{84}+\frac{241}{2268000} v^{4}+\frac{22871}{6123600000} v^{6} \\
& +\frac{112778137}{2728063800000000} v^{8} \\
& +\frac{13599351683}{55857106305000000000} v^{10}+\cdots, \\
& b_{1}^{\prime \prime}=\frac{1}{24}+\frac{1}{21600} v^{4}+\frac{167}{58320000} v^{6} \\
& +\frac{528389}{16533720000000} v^{8} \\
& +\frac{967343}{5845851000000000} v^{10}+\cdots \\
& b_{2}^{\prime \prime}=\frac{125}{336}-\frac{1}{21600} v^{4}-\frac{2867}{408240000} v^{6} \\
& -\frac{3022109}{16533720000000} v^{8} \\
& -\frac{1457821}{730731375000000} v^{10}+\cdots, \\
& \widehat{a}_{21}=\frac{1}{50}+\frac{1}{15000} v^{2}+\frac{1}{11250000} v^{4} \\
& +\frac{1}{15750000000} v^{6}+\frac{1}{35437500000000} v^{8} \\
& +\frac{1}{116943750000000000} v^{10}+\cdots, \\
& \widehat{a}_{32}=\frac{7}{27}+\frac{37}{12150} v^{2}+\frac{287}{6561000} v^{4} \\
& -\frac{68827}{516678750000} v^{6} \\
& +\frac{7097417}{1674039150000000} v^{8} \\
& -\frac{39720321233}{621487034437500000000} v^{10}+\cdots, \\
& \widehat{a}_{43}=\frac{9}{35}-\frac{43}{3000} v^{2}+\frac{3323}{1350000} v^{4}-\frac{111136579}{255150000000} v^{6} \\
& +\frac{26973882539}{344452500000000} v^{8} \\
& -\frac{721361598388001}{51151196250000000000} v^{10}+\cdots, \\
& a_{32}=\frac{289}{4860}-\frac{67}{729000} v^{2}+\frac{26141}{2755620000} v^{4} \\
& -\frac{4671541}{39858075000000} v^{6}
\end{aligned}
$$

$$
\begin{aligned}
& +\frac{2216008103}{1104865839000000000} v^{8} \\
& -\frac{15664491766661}{484759886861250000000000} v^{10}+\cdots, \\
& a_{43}=\frac{7}{150}-\frac{221}{135000} v^{2}+\frac{114463}{637875000} v^{4} \\
& -\frac{1010472889}{34445250000000} v^{6} \\
& +\frac{222576819697}{42625996875000000} v^{8} \\
& -\frac{84318883418716333}{89770349418750000000000} v^{10}+\cdots, \\
& \gamma_{2}=1+\frac{1}{15000} v^{4}+\frac{1}{11250000} v^{6}+\frac{1}{15750000000} v^{8} \\
& +\frac{1}{35437500000000} v^{10}+\cdots, \\
& \gamma_{3}=1-\frac{59}{225} v^{2}-\frac{9569}{1215000} v^{4}-\frac{74831}{911250000} v^{6} \\
& +\frac{26104429}{11481750000000} v^{8} \\
& -\frac{495937229}{25833937500000000} v^{10}+\cdots \\
& \gamma_{4}=1-v^{2}+\frac{19}{1000} v^{4}+\frac{2267}{4050000} v^{6} \\
& -\frac{4858267}{255150000000} v^{8} \\
& +\frac{65060629}{344452500000000} v^{10}+\cdots \text {, } \\
& \widehat{\gamma}_{2}=1+\frac{1}{150} v^{2}+\frac{1}{75000} v^{4}+\frac{1}{78750000} v^{6} \\
& +\frac{1}{141750000000} v^{8} \\
& +\frac{1}{389812500000000} v^{10}+\cdots, \\
& \widehat{\gamma}_{3}=1-\frac{1}{270} v^{2}+\frac{13}{60750} v^{4}-\frac{2032}{717609375} v^{6} \\
& +\frac{136109}{2906317968750} v^{8} \\
& -\frac{2726807}{3596568486328125} v^{10}+\cdots, \\
& \widehat{\gamma}_{4}=1+\frac{1}{150} v^{2}+\frac{79}{15000} v^{4}-\frac{2161951}{2126250000} v^{6} \\
& +\frac{78384961}{425250000000} v^{8} \\
& -\frac{5664933289891}{170503987500000000} v^{10}+\cdots
\end{aligned}
$$

where $\gamma_{1}=1, \widehat{\gamma}_{1}=1$. 
This results in the new method called EFMRKT5. As $v \longrightarrow 0$, the coefficients $b_{1}, b_{2}, b_{1}^{\prime}, b_{2}^{\prime}, b_{2}^{\prime \prime}, b_{2}^{\prime \prime}, a_{32}, a_{42}, \widehat{a}_{21}$, $\widehat{a}_{32}, \widehat{a}_{43}, \gamma_{2}, \gamma_{3}, \gamma_{4}, \widehat{\gamma}_{2}, \widehat{\gamma}_{3}$, and $\widehat{\gamma}_{4}$ of the new method EFMRKT5 reduce to the coefficients of the original method RKT5. That is to say, $b_{1}(0), b_{2}(0), b_{1}^{\prime}(0), b_{2}^{\prime}(0), b_{2}^{\prime \prime}(0), b_{2}^{\prime \prime}(0), a_{32}(0), a_{42}(0)$, $\widehat{a}_{21}(0), \widehat{a}_{32}(0), \widehat{a}_{43}(0), \gamma_{2}(0), \gamma_{3}(0), \gamma_{4}(0), \widehat{\gamma}_{2}(0), \widehat{\gamma}_{3}(0)$, and $\widehat{\gamma}_{4}(0)$ are identical to $b_{1}, b_{2}, b_{1}^{\prime}, b_{2}^{\prime}, b_{2}^{\prime \prime}, b_{2}^{\prime \prime}, a_{32}, a_{42}, \widehat{a}_{21}, \widehat{a}_{32}, \widehat{a}_{43}, \gamma_{2}, \gamma_{3}$, $\gamma_{4}, \widehat{\gamma}_{2}, \widehat{\gamma}_{3}$, and $\widehat{\gamma}_{4}$ of RKT5 method. Other than that, $v \longrightarrow 0$, as EFMRKT5 method will have the same error constant as RKT5 method.

3.2. Trigonometrically Fitted MRKT Method. Exponentially fitted method leads to trigonometrically fitted method when replacing $v=w h$ with $i v$ and solving (8) to (9) to find $a_{i, i-1}$, $\widehat{a}_{i, i-1}, \gamma_{i}$, and $\widehat{\gamma}_{i}$.

$$
\begin{aligned}
\gamma_{i} & =\cos \left(v c_{i}\right)-\frac{1}{2} v^{2} c_{i}^{2}-v^{3} \sum_{j=1}^{i-1} a_{i, j} \sin \left(v c_{j}\right), \\
\widehat{a}_{i, i-1} & =\frac{1-\cos \left(v c_{i}\right)-v^{2} \sum_{j=1}^{i-2} \widehat{a}_{i, j} \cos \left(v c_{j}\right)}{v^{2} \cos \left(v c_{i-1}\right)}, \\
a_{i, i-1} & =\frac{-\sin \left(v c_{i}\right)+v \cdot c_{i}-v^{3} \sum_{j=1}^{i-2} a_{i, j} \cos \left(v c_{j}\right)}{v^{3} \cos \left(v c_{i-1}\right)}, \\
\widehat{\gamma}_{i} & =\frac{\sin \left(v c_{i}\right)+v^{2} \sum_{j=1}^{i-2} \widehat{a}_{i, j} \sin \left(v c_{j}\right)}{v c_{i}}, \quad i=2, \ldots, s .
\end{aligned}
$$

Consider the same coefficients of fifth-order four-stage method developed by Fawzi et al.[15] as in Section 3.1. Solving the (31) to (34) and letting $\widehat{a}_{21}, \widehat{a}_{32}, \widehat{a}_{43}, a_{32}, a_{43}, \gamma_{2}, \gamma_{3}, \gamma_{4}, \widehat{\gamma}_{2}$, $\widehat{\gamma}_{3}$, and $\widehat{\gamma}_{4}$ be free parameters will give

$$
\begin{aligned}
\widehat{a}_{21} & =\frac{1-\cos (v / 5)}{v^{2}}, \\
\widehat{a}_{32} & =\frac{1-\cos (2 v / 3)+(1 / 27) v^{2}}{v^{2} \cos (v / 5)}, \\
\widehat{a}_{43} & =\frac{1-\cos (v)-v^{2}(3 / 10-(2 / 35) \cos (v / 5))}{v^{2} \cos (2 v / 3)}, \\
a_{32}= & \frac{-\sin (2 v / 3)+2 v / 3+(49 / 4860) v^{3}}{v^{3} \cos (v / 5)}, \\
a_{43}= & \frac{v-\sin (v)-v^{3}(7 / 50-(1 / 50) \cos (v / 5))}{v^{3} \cos (2 v / 3)}, \\
\gamma_{2}= & \cos \left(\frac{v}{5}\right)-\frac{v^{2}}{50}, \\
\gamma_{3}= & \cos \left(\frac{2 v}{3}\right)-\frac{2 v^{2}}{9}-\frac{301 v^{3}}{4860} \sin \left(\frac{v}{5}\right), \\
\gamma_{4}= & \cos (v)-\frac{v^{2}}{2}-v^{3}\left(-\frac{1}{50} \sin \left(\frac{v}{5}\right)+\frac{1}{25} \sin \left(\frac{2 v}{3}\right)\right), \\
\widehat{\gamma}_{2}= & \frac{5}{v} \sin \left(\frac{v}{5}\right), \\
\widehat{\gamma}_{3}= & \frac{2}{3 v} \sin \left(\frac{2 v}{3}\right)+\frac{2\left(1-\cos (2 v / 3)+v^{2} / 27\right) \sin (v / 5)}{3 v^{2} \cos (v / 35)}, \\
& \frac{2 v}{35} \sin \left(\frac{v}{5}\right)
\end{aligned} .
$$

Next, solving (10) to (12), and using the above Fawzi coefficients to find $b_{1}, b_{2}, b_{1}^{\prime}, b_{2}^{\prime}, b_{1}^{\prime \prime}$, and $b_{2}^{\prime \prime}$,

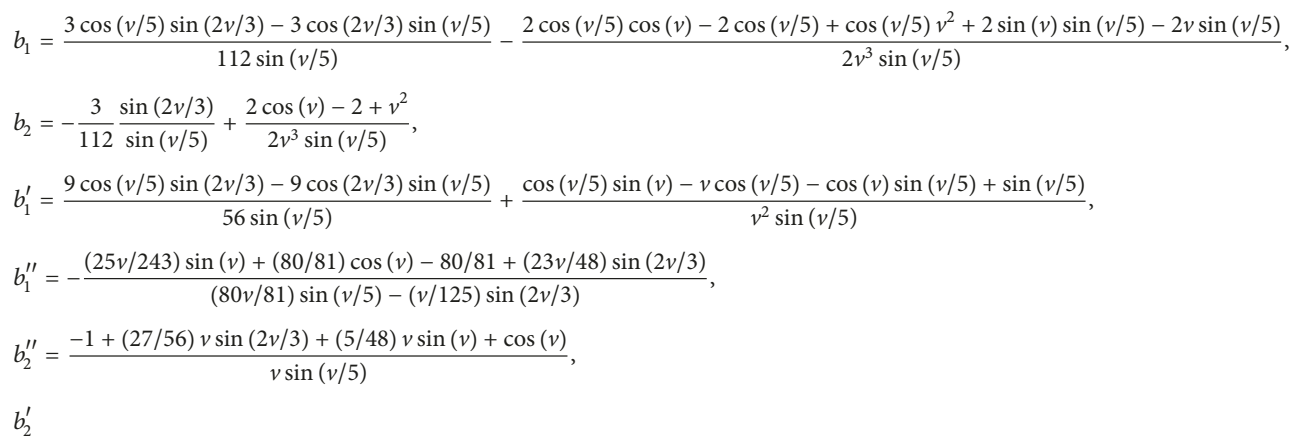

These lead to our new explicit trigonometrically fitted MRKT which is called TFMRKT5 method. The corresponding Taylor series expansion of the solution is given by

$$
\begin{aligned}
b_{1}= & \frac{1}{48}-\frac{1}{2160} v^{2}+\frac{101}{136080000} v^{4} \\
& +\frac{5713}{183708000000} v^{6}
\end{aligned}
$$

$$
\begin{aligned}
& -\frac{11330339}{81841914000000000} v^{8} \\
& +\frac{57722879}{134057055132000000000} v^{10}+\cdots, \\
b_{2}= & \frac{5}{42}+\frac{1}{2160} v^{2}-\frac{2921}{136080000} v^{4} \\
& +\frac{1361}{45927000000} v^{6}
\end{aligned}
$$




$$
\begin{aligned}
& +\frac{54293587}{40920957000000000} v^{8} \\
& +\frac{721361598388001}{51151196250000000000} v^{10}+\cdots, \\
& -\frac{6964030429}{670285275660000000000} v^{10}+\cdots, \\
& b_{1}^{\prime}=\frac{1}{24}-\frac{17}{283500} v^{4}+\frac{149}{218700000} v^{6} \\
& -\frac{1055069}{341007975000000} v^{8} \\
& +\frac{23025689}{2538959377500000000} v^{10}+\cdots, \\
& b_{2}^{\prime}=\frac{25}{84}+\frac{241}{2268000} v^{4}-\frac{22871}{6123600000} v^{6} \\
& +\frac{112778137}{2728063800000000} v^{8} \\
& -\frac{13599351683}{55857106305000000000} v^{10}+\cdots, \\
& b_{1}^{\prime \prime}=\frac{1}{24}+\frac{1}{21600} v^{4}-\frac{167}{58320000} v^{6} \\
& +\frac{528389}{16533720000000} v^{8} \\
& -\frac{967343}{5845851000000000} v^{10}+\cdots, \\
& b_{2}^{\prime \prime}=\frac{125}{336}-\frac{1}{21600} v^{4}+\frac{2867}{408240000} v^{6} \\
& -\frac{3022109}{16533720000000} v^{8} \\
& +\frac{1457821}{730731375000000} v^{10}+\cdots, \\
& \widehat{a}_{21}=\frac{1}{50}-\frac{1}{15000} v^{2}+\frac{1}{11250000} v^{4} \\
& -\frac{1}{15750000000} v^{6}+\frac{1}{35437500000000} v^{8} \\
& -\frac{1}{116943750000000000} v^{10}+\cdots, \\
& \widehat{a}_{32}=\frac{7}{27}-\frac{37}{12150} v^{2}+\frac{287}{6561000} v^{4} \\
& +\frac{68827}{516678750000} v^{6} \\
& +\frac{7097417}{1674039150000000} v^{8} \\
& +\frac{39720321233}{621487034437500000000} v^{10}+\cdots, \\
& \widehat{a}_{43}=\frac{9}{35}+\frac{43}{3000} v^{2}+\frac{3323}{1350000} v^{4}+\frac{111136579}{255150000000} v^{6} \\
& +\frac{26973882539}{344452500000000} v^{8} \\
& \begin{aligned}
a_{32}= & \frac{289}{4860}+\frac{67}{729000} v^{2}+\frac{26141}{2755620000} v^{4} \\
& +\frac{4671541}{39858075000000} v^{6} \\
& +\frac{2216008103}{1104865839000000000} v^{8} \\
& +\frac{15664491766661}{484759886861250000000000} v^{10}+\cdots,
\end{aligned} \\
& a_{43}=\frac{7}{150}+\frac{221}{135000} v^{2}+\frac{114463}{637875000} v^{4} \\
& +\frac{1010472889}{34445250000000} v^{6} \\
& +\frac{222576819697}{42625996875000000} v^{8} \\
& +\frac{84318883418716333}{89770349418750000000000} v^{10}+\cdots, \\
& \gamma_{2}=1-\frac{1}{25} v^{2}+\frac{1}{15000} v^{4}-\frac{1}{11250000} v^{6} \\
& +\frac{1}{15750000000} v^{8}-\frac{1}{35437500000000} v^{10} \\
& +\cdots \text {, } \\
& \gamma_{3}=1-\frac{59}{225} v^{2}-\frac{9569}{1215000} v^{4}-\frac{74831}{911250000} v^{6} \\
& +\frac{26104429}{11481750000000} v^{8} \\
& -\frac{495937229}{25833937500000000} v^{10}+\cdots, \\
& \gamma_{4}=1-v^{2}+\frac{19}{1000} v^{4}+\frac{2267}{4050000} v^{6} \\
& -\frac{4858267}{255150000000} v^{8} \\
& +\frac{65060629}{344452500000000} v^{10}+\cdots \text {, } \\
& \widehat{\gamma}_{2}=1-\frac{1}{150} v^{2}+\frac{1}{75000} v^{4}-\frac{1}{78750000} v^{6} \\
& +\frac{1}{141750000000} v^{8} \\
& -\frac{1}{389812500000000} v^{10}+\cdots \text {, } \\
& \widehat{\gamma}_{3}=1+\frac{1}{270} v^{2}+\frac{13}{60750} v^{4}+\frac{2032}{717609375} v^{6} \\
& +\frac{136109}{2906317968750} v^{8}
\end{aligned}
$$




$$
\begin{gathered}
+\frac{2726807}{3596568486328125} v^{10}+\cdots, \\
\widehat{\gamma}_{4}=1-\frac{1}{150} v^{2}+\frac{79}{15000} v^{4}+\frac{2161951}{2126250000} v^{6} \\
+\frac{78384961}{425250000000} v^{8} \\
+\frac{5664933289891}{170503987500000000} v^{10}+\cdots,
\end{gathered}
$$

where $\gamma_{1}=1, \widehat{\gamma}_{1}=1$.

This results in the new method called TFMRKT5. As $v \longrightarrow 0$, the coefficients $b_{1}, b_{2}, b_{1}^{\prime}, b_{2}^{\prime}, b_{2}^{\prime \prime}, b_{2}^{\prime \prime}, a_{32}, a_{42}, \widehat{a}_{21}, \widehat{a}_{32}$, $\widehat{a}_{43}, \gamma_{2}, \gamma_{3}, \gamma_{4}, \widehat{\gamma}_{2}, \widehat{\gamma}_{3}$, and $\widehat{\gamma}_{4}$ of the new method TFMRKT5 reduce to the coefficients of the original method RKT5. That is to say, $b_{1}(0), b_{2}(0), b_{1}^{\prime}(0), b_{2}^{\prime}(0), b_{2}^{\prime \prime}(0), b_{2}^{\prime \prime}(0), a_{32}(0), a_{42}(0)$, $\widehat{a}_{21}(0), \widehat{a}_{32}(0), \widehat{a}_{43}(0), \gamma_{2}(0), \gamma_{3}(0), \gamma_{4}(0), \widehat{\gamma}_{2}(0), \widehat{\gamma}_{3}(0)$, and $\widehat{\gamma}_{4}(0)$ are identical to $b_{1}, b_{2}, b_{1}^{\prime}, b_{2}^{\prime}, b_{2}^{\prime \prime}, b_{2}^{\prime \prime}, a_{32}, a_{42}, \widehat{a}_{21}, \widehat{a}_{32}, \widehat{a}_{43}, \gamma_{2}, \gamma_{3}$, $\gamma_{4}, \widehat{\gamma}_{2}, \widehat{\gamma}_{3}$, and $\widehat{\gamma}_{4}$ of RKT5 method. Other than that, $v \longrightarrow 0$, as TFMRKT5 method will have the same error constant as RKT5 method.

\section{Error Analysis}

In this section, we will find the principal local truncation errors for $y, y^{\prime}$, and $y^{\prime \prime}$ (i.e., $\tau_{n+1}, \tau_{n+1}^{\prime}, \tau_{n+1}^{\prime \prime}$ ) of the new exponentially fitted and trigonometrically fitted explicit modified Runge-Kutta type methods, respectively. We first find the Taylor series expansion of the actual solution $y\left(x_{n}+h\right)$, the first derivative of the actual solution $y^{\prime}\left(x_{n}+h\right)$, and the second derivative of the actual solution $y^{\prime \prime}\left(x_{n}+h\right)$, the approximate solution $y_{n+1}$, the first derivative of the approximate solution $y_{n+1}^{\prime}$, and the second derivative of the approximate solution $y_{n+1}^{\prime \prime}$. The local truncation errors of $y, y^{\prime}$, and $y^{\prime \prime}$ are given as

$$
\begin{gathered}
\tau_{n+1}=y_{n+1}-y\left(x_{n}+h\right), \\
\tau_{n+1}^{\prime}=y_{n+1}^{\prime}-y^{\prime}\left(x_{n}+h\right), \\
\tau_{n+1}^{\prime \prime}=y_{n+1}^{\prime \prime}-y^{\prime \prime}\left(x_{n}+h\right)
\end{gathered}
$$

The $\tau_{n+1}, \tau_{n+1}^{\prime}$, and $\tau_{n+1}^{\prime \prime}$ of the methods are given in the Appendix.

Notes: from $\tau_{n+1}, \tau_{n+1}^{\prime}$, and $\tau_{n+1}^{\prime \prime}$, we can see that the order of TFMRKT5 is order 5 because all of the coefficients up to $h^{5}$ vanished.

\section{Problems Tested and Numerical Results}

In this section, we will apply the new explicit exponentially fitted modified Runge-Kutta type method to some $y^{\prime \prime \prime}=$ $f\left(x, y, y^{\prime}\right)$ ODEs for problems (1)-(4) which consist of exponential solutions and the new trigonometrically fitted modified Runge-Kutta type method to some ODEs problems (5)-(8) with trigonometric functions solutions. The numerical results are compared with the results obtained when the same set of problems are reduced to a system of first-order equations and is solved using the existing Runge-Kutta of the same order.

(i) $h$ : step sizes.

(ii) TFMRKT5: the four-stage fifth-order trigonometrically fitted RK type method derived in this paper.

(iii) EFMRKT5: the four-stage fifth-order exponentially fitted RK type method derived in this paper.

(iv) RKT5: the four-stage fifth-order RK type method given by Fawzi et al. [15].

(v) RK5B: the six-stage fifth-order RK method given in Butcher [16].

(vi) RKF5: the six-stage fifth-order RK method given in Lambert [17].

(vii) TFRK: the six-stage fifth-order trigonometrically fitted RK method given in Anastassi et al. [18].

Problem 2 (homogeneous linear problem).

$$
\begin{aligned}
& y^{\prime \prime \prime}(x)=2 y^{\prime}(x), \\
& y(0)=0, y^{\prime}(0)=1, y^{\prime \prime}(0)=0,
\end{aligned}
$$

exact solution is

$$
y(x)=\frac{\sqrt{2} e^{\sqrt{2} x}}{4}-\frac{\sqrt{2} e^{-\sqrt{2} x}}{4}
$$

Estimated frequency $\omega=\sqrt{2}$.

Problem 3 ( homogeneous linear system).

$$
\begin{aligned}
& y_{1}^{\prime \prime \prime}(x)=8 y_{3}^{\prime}(x), \\
& y_{1}(0)=2, y_{1}^{\prime}(0)=4, y_{1}^{\prime \prime}(0)=8, \\
& y_{2}^{\prime \prime \prime}(x)=8 y_{1}^{\prime}(x), \\
& y_{2}(0)=4, y_{2}^{\prime}(0)=8, y_{2}^{\prime \prime}(0)=16, \\
& y_{3}^{\prime \prime \prime}(x)=y_{2}^{\prime}(x), \quad \\
& y_{3}(0)=1, y_{3}^{\prime}(0)=2, y_{3}^{\prime \prime}(0)=4,
\end{aligned}
$$

exact solutions are

$$
\begin{aligned}
& y_{1}(x)=2 e^{2 x} \\
& y_{2}(x)=4 e^{2 x} \\
& y_{3}(x)=e^{2 x}
\end{aligned}
$$

Estimated frequency $\omega=2$. 
Problem 4 ( inhomogeneous linear system ).

$$
\begin{aligned}
& y_{1}^{\prime \prime \prime}(x)=y_{3}^{\prime}(x)+1, \\
& y_{1}(0)=2, y_{1}^{\prime}(0)=3, y_{1}^{\prime \prime}(0)=5, \\
& y_{2}^{\prime \prime \prime}(x)=y_{1}^{\prime}(x)+2, \\
& y_{2}(0)=1, y_{2}^{\prime}(0)=2, y_{2}^{\prime \prime}(0)=5, \\
& y_{3}^{\prime \prime \prime}(x)=y_{2}^{\prime}(x)+3, \\
& y_{3}(0)=0, y_{3}^{\prime}(0)=4, y_{3}^{\prime \prime}(0)=5,
\end{aligned}
$$

exact solutions are

$$
\begin{aligned}
& y_{1}(x)=5 e^{x}-2 x-3, \\
& y_{2}(x)=5 e^{x}-3 x-4, \\
& y_{3}(x)=5 e^{x}-x-5 .
\end{aligned}
$$

Estimated frequency $\omega=1$.

Problem 5 (inhomogeneous linear problem).

$$
y^{\prime \prime \prime}(x)=5 y^{\prime}(x)+\sinh (x),
$$

$$
y(0)=-\frac{1}{4}, y^{\prime}(0)=0, y^{\prime \prime}(0)=-\frac{1}{4},
$$

exact solution is

$$
y(x)=-\frac{e^{x}}{8}-\frac{e^{-x}}{8} .
$$

Estimated frequency $\omega=1$.

Problem 6 (homogeneous linear problem).

$$
\begin{aligned}
& y^{\prime \prime \prime}(x)=-25 y^{\prime}(x), \\
& y(0)=0, y^{\prime}(0)=0, y^{\prime \prime}(0)=1,
\end{aligned}
$$

exact solution is

$$
y(x)=\frac{1}{25}-\frac{1}{25} \cos (5 x) .
$$

Estimated frequency $\omega=5$.

Problem 7 (inhomogeneous linear problem).

$$
\begin{aligned}
& y^{\prime \prime \prime}(x)=-27 y^{\prime}(x)+\sin (x), \\
& y(0)=1, y^{\prime}(0)=-1, y^{\prime \prime}(0)=0,
\end{aligned}
$$

exact solution is

$$
\begin{aligned}
y(x)= & \frac{\sqrt{3}}{702} \cos (3 \sqrt{3} x)-\frac{\sqrt{3}}{9} \sin (3 \sqrt{3} x) \\
& -\frac{1}{26} \cos (x)+\frac{28}{27} .
\end{aligned}
$$

Estimated frequency $\omega=3 \sqrt{3}$.
Problem 8 (inhomogeneous linear system).

$$
\begin{array}{r}
y_{1}^{\prime \prime \prime}(x)=-27 y_{1}^{\prime}(x), \\
y_{1}(0)=0, \quad y_{1}^{\prime}(0)=-1, y_{1}^{\prime}(0)=0, \\
y_{2}^{\prime \prime \prime}(x)=-27 y_{2}^{\prime}(x)+\cos (x), \\
y_{2}(0)=1, y_{2}^{\prime}(0)=-1, y_{2}^{\prime \prime}(0)=0, \\
y_{3}^{\prime \prime \prime}(x)=-27 y_{3}^{\prime}(x), \\
y_{3}(0)=1, y_{3}^{\prime}(0)=0, y_{3}^{\prime \prime}(0)=-1,
\end{array}
$$

exact solutions are

$$
\begin{aligned}
& y_{1}(x)=-\frac{\sqrt{3}}{9} \sin (3 \sqrt{3} x), \\
& y_{2}(x)=1-\frac{3 \sqrt{3}}{26} \sin (3 \sqrt{3} x)+\frac{1}{26} \sin (x), \\
& y_{3}(x)=\frac{26}{27}+\frac{1}{27} \cos (3 \sqrt{3} x) .
\end{aligned}
$$

Estimated frequency $\omega=3 \sqrt{3}$.

Problem 9 (inhomogeneous linear system).

$$
\begin{aligned}
& y_{1}^{\prime \prime \prime}(x)=-7 y_{2}^{\prime}(x)-\cos (x), \\
& y_{1}(0)=\frac{1}{7}, y_{1}^{\prime}(0)=0, y_{1}^{\prime \prime}(0)=-1, \\
& y_{2}^{\prime \prime \prime}(x)=-7 y_{1}^{\prime}(x)-\cos (x), \\
& y_{2}(0)=1, y_{2}^{\prime}(0)=0, y_{2}^{\prime \prime}(0)=-1, \\
& y_{3}^{\prime \prime \prime}(x)=-7 y_{3}^{\prime}(x)-\cos (x), \\
& y_{3}(0)=0, y_{3}^{\prime}(0)=1, y_{3}^{\prime \prime}(0)=0,
\end{aligned}
$$

exact solutions are

$$
\begin{aligned}
y_{1}(x)= & -\frac{1}{6} \sin (x)+\frac{\sqrt{7}}{42} \sin (\sqrt{7} x)+\frac{1}{7} \cos (\sqrt{7} x), \\
y_{2}(x)= & -\frac{1}{6} \sin (x)+\frac{\sqrt{7}}{42} \sin (x) \sqrt{7}+\frac{6}{7} \\
& +\frac{1}{7} \cos (\sqrt{7} x), \\
y_{3}(x)= & \frac{\sqrt{7}}{6} \sin (\sqrt{7} x)-\frac{1}{6} \sin (x) .
\end{aligned}
$$

Estimated frequency $\omega=1$.

\section{An Application to a Problem in Thin Film Flow}

Here, we will use the suggested method to a famous problem in engineering and physics based on the thin film flow 
of a liquid. Many researchers in the literature explain this problem more. Momoniat and Mahomed[19] constructed symmetry reduction and numerical solution of a third-order ODE from thin film flow. Tuck and Schwartz [20] discussed the movement of a thin film of viscous fluid over a solid surface and taken into account tension and gravity, as well as viscosity. The problem was evaluated and solved using thirdorder ODE as follows:

$$
\frac{d^{3} y}{d x^{3}}=f(y) .
$$

Many forms of the function were studied by [20] for the drainage dry surface; it has the form of $f(y)$ which can be stated as

$$
\frac{d^{3} y}{d x^{3}}=-1+\frac{1}{y^{2}}
$$

When the surface is prewetted by a thin film with thickness $\delta>0$ (where $\delta>0$ is very small), the function $f$ is given by

$$
f(y)=-1+\frac{1+\delta+\delta^{2}}{y^{2}}-\frac{\delta+\delta^{2}}{y^{3}}
$$

Problems concerning the flow of thin films of viscous fluid with a free surface in which surface tension effects play a role typically lead to third-order ODEs governing the shape of the free surface of the fluid, $y=y(x)$. As indicated by [20], one such equation is

$$
y^{\prime \prime \prime}(x)=y^{-k}, \quad x \geq x_{0}
$$

with initial conditions

$$
\begin{gathered}
y\left(x_{0}\right)=y_{0}, \\
y^{\prime}\left(x_{0}\right)=y_{0}^{\prime}, \\
y^{\prime \prime}\left(x_{0}\right)=y_{0}^{\prime \prime},
\end{gathered}
$$

where $y_{0}, y_{0}^{\prime}$, and $y_{0}^{\prime \prime}$ are constants, which is of specific significance since it portrays the dynamic balance amongst surface and gooey strengths in a thin fluid layer in disregard of gravity. For compare and contrast, we utilized RungeKutta methods which are fifth-order (RKT5, RK5B, RKF5, and TFRKT) strategies, individually. To utilize Runge-Kutta techniques we write (1) as a system of three first-order equations. Biazar et al. [21] we can write (58) as the following system:

$$
\begin{aligned}
& \frac{d y_{1}}{d x}=y_{2}(x), \\
& \frac{d y_{2}}{d x}=y_{3}(x), \\
& \frac{d y_{2}}{d x}=y_{1}^{-k},
\end{aligned}
$$

where

$$
\begin{aligned}
& y_{1}(0)=1, \\
& y_{2}(0)=1, \\
& y_{3}(0)=1 .
\end{aligned}
$$

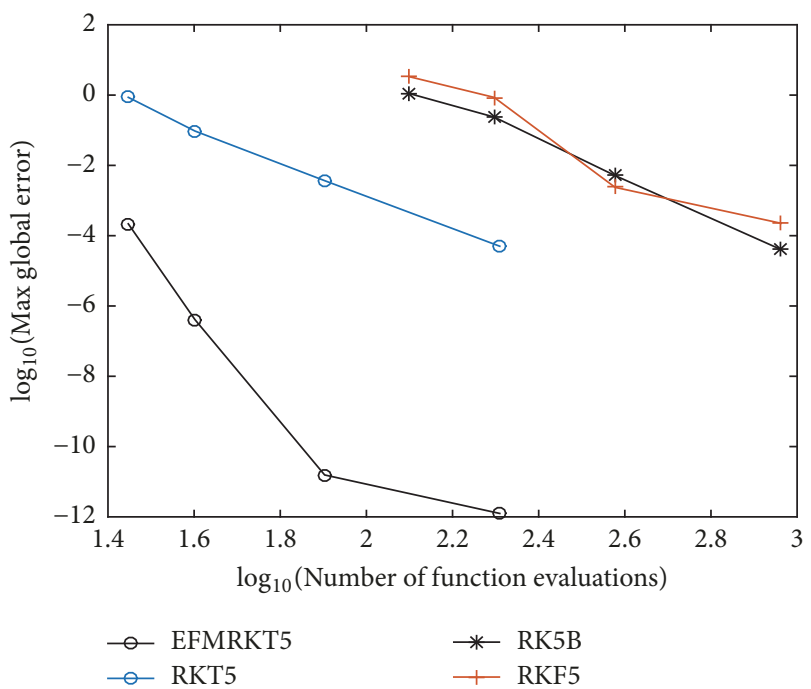

FIgURE 1: The efficiency curve for EFMRKT5, RKT5, RK5B, and RKF5 for Problem 2 with $x_{\text {end }}=5$ and $h=0.1,0.25,0.5,0.75$.

We have taken $x_{0}=0$ and $y_{0}=y_{0}^{\prime}=y_{0}^{\prime \prime}=1$. Unfortunately, for general $k$, (58) cannot be solved analytically. However, we can use these reductions to determine an efficient way to solve (1) numerically. Here, we are focusing on the cases $k=2$ and $k=3$ (see Mechee et al.[22]).

\section{Discussion and Conclusion}

In this research, we have derived exponentially fitted and trigonometrically fitted explicit modified Runge-Kutta type methods for solving $y^{\prime \prime \prime}(x)=f\left(x, y, y^{\prime}\right)$ with application to thin film flow problem. Consequently, the new four-stage fifth-order exponentially-fitted and trigonometrically-fitted methods which are denoted as EFMRKT5 and TFMRKT5, respectively, were constructed and we used in numerical comparison the criteria based on computing the maximum error in the solution $\left(\max \left(\left|y\left(t_{n}\right)-y_{n}\right|\right)\right)$ which is equal to the maximum between absolute errors of the actual solutions and computed solutions. The numerical outcomes are plotted in Figures 1-8. Figures 1-8 demonstrate that the new TFMRKT5 and EFMRKT5 methods require less capacity assessments than the RKT5, RK5B, RKF5, and TFRK methods. The figures showed the efficiency of the new methods where the common logarithm of the maximum global error throughout the integration versus computational cost was measured by the number of function evaluations. The numerical results obtained showed clearly that the global error for a short period of integration for the new exponentially fitted method and for a large period of integration for the new trigonometrically fitted explicit modified Runge-Kutta type method is smaller than that of the other existing methods. The new EFMRKT5 and TFMRKT5 methods are much more efficient than the other existing methods when solving third-order ODEs of the form $y^{\prime \prime \prime}=f\left(x, y, y^{\prime}\right)$ straightforwardly. For Tables 2 and 3 we observed that the numerical results using TFMRKT5 and EFMRKT5 methods are correct to five decimal places. Applying RK5B, RKF5, TFRK, and RKT5 to 
TABLE 2: Numerical results for problem in Thin Film Flow (58) taking $h=0.1$ and $k=2$.

\begin{tabular}{lccccccc}
\hline$x$ & Exact Solution & RK5B & RKF5 & RKT5 & EMFRKT5 & TFRK & TFMRKT5 \\
\hline 0.0 & 1.000000000 & 1.000000000 & 1.000000000 & 1.000000000 & 1.000000000 & 1.000000000 & 1.000000000 \\
0.2 & 1.221211030 & 1.2212100068 & 1.2212100097 & 1.2212100039 & 1.2212100052 & 1.2212100218 & 1.2212100052 \\
0.4 & 1.488834893 & 1.4888347851 & 1.4888347895 & 1.4888347797 & 1.4888347885 & 1.4888348090 & 1.4888347885 \\
0.6 & 1.807361404 & 1.8073614063 & 1.8073614114 & 1.8073613988 & 1.8073614237 & 1.8073614357 & 1.8073614237 \\
0.8 & 2.179819234 & 2.1798192463 & 2.1798192513 & 2.1798192371 & 2.1798192873 & 2.1798192788 & 2.1798192873 \\
1.0 & 2.608275822 & 2.6082748841 & 2.6082748883 & 2.6082748735 & 2.6082749587 & 2.6082749176 & 2.6082749587 \\
\hline
\end{tabular}

TABLE 3: Numerical results for problem in thin film flow (58) taking $h=0.01$ and $k=2$.

\begin{tabular}{lccccccc}
\hline$x$ & Exact Solution & RK5B & RKF5 & RKT5 & EFMRKT5 & TFRK & TFMRKT5 \\
\hline 0.0 & 1.000000000 & 1.000000000 & 1.000000000 & 1.000000000 & 1.000000000 & 1.000000000 & 1.000000000 \\
0.2 & 1.221211030 & 1.2212100045 & 1.2212100045 & 1.2212100045 & 1.2212100045 & 1.2212100045 & 1.2212100045 \\
0.4 & 1.488834893 & 1.4888347799 & 1.4888347799 & 1.4888347799 & 1.4888347799 & 1.4888347799 & 1.4888347799 \\
0.6 & 1.807361404 & 1.8073613977 & 1.8073613977 & 1.8073613977 & 1.8073613977 & 1.8073613977 & 1.8073613977 \\
0.8 & 2.179819234 & 2.1798192339 & 2.1798192339 & 2.1798192339 & 2.1798192340 & 2.1798192339 & 2.1798192340 \\
1.0 & 2.608275822 & 2.6082748676 & 2.6082748676 & 2.6082748676 & 2.6082748677 & 2.6082748676 & 2.6082748677 \\
\hline
\end{tabular}

(58) for $k=2$ also yields five-decimal place accuracy. Tables 4 and 5 show the numerical results for the case $k=3$ with $h=0.1$ and $h=0.01$ since for $k=3$, Problem (58) cannot be solved analytically. Table 4 shows that TFMRKT5 and EFMRKT5 manage to achieve the numerical results which agree to seven decimal places when compared to RK5B, RKF5, TFRK, and RKT5 for $h=0.1$. In Table 5 the numerical results for TFMRKT5 and EFMRKT5 agree to nine decimal places when compared to RK5B, RKF5, TFRK, and RKT5 for $h=0.01$. For Table 7 we observe that RK5B, RKF5, RKT5, TFRK, TFMRKT5, and EFMRKT5 have similar order of accuracy. In Table 6 values of the error are different. Therefore it is consistent with results displayed in Tables 2 and 3. Figures 9 and 10 show that the new EFMRKT5 and TFMRKT5 methods require less function evaluations than the RK5B, RKF5, TFRK, and RKT5 methods. This is because when problem (58) is solved using RK5B, RKF5, TFRK, and RKT5 methods, it needs to be reduced to a system of firstorder equations which is three times the dimension.

\section{Appendix}

The principal local truncation errors for $y, y^{\prime}$, and $y^{\prime \prime}$ (i.e., $\left.\tau_{n+1}, \tau_{n+1}^{\prime}, \tau_{n+1}^{\prime \prime}\right)$ for EFMRKT5 are as follows:

$$
\begin{aligned}
\tau_{n+1} & =\left(-\frac{1}{3600} y_{x x}{ }^{2} F y z-\frac{1}{10800} y_{x}^{3} F y y y\right. \\
- & \frac{1}{3600} y_{x} F y y y_{x x}+\frac{527}{162000} y_{x} F y z F \\
+ & \frac{527}{162000} F z z y_{x x} F-\frac{1}{1800} y_{x} F x y z y_{x x} \\
& -\frac{1}{3600} y_{x}^{2} F z y y y_{x x}-\frac{1}{3600} y_{x} y_{x x}{ }^{2} F z y z \\
& +\frac{971}{907200} F z F y y_{x}-\frac{1}{10800} F x x x+\frac{1}{10800} w^{2} F x
\end{aligned}
$$

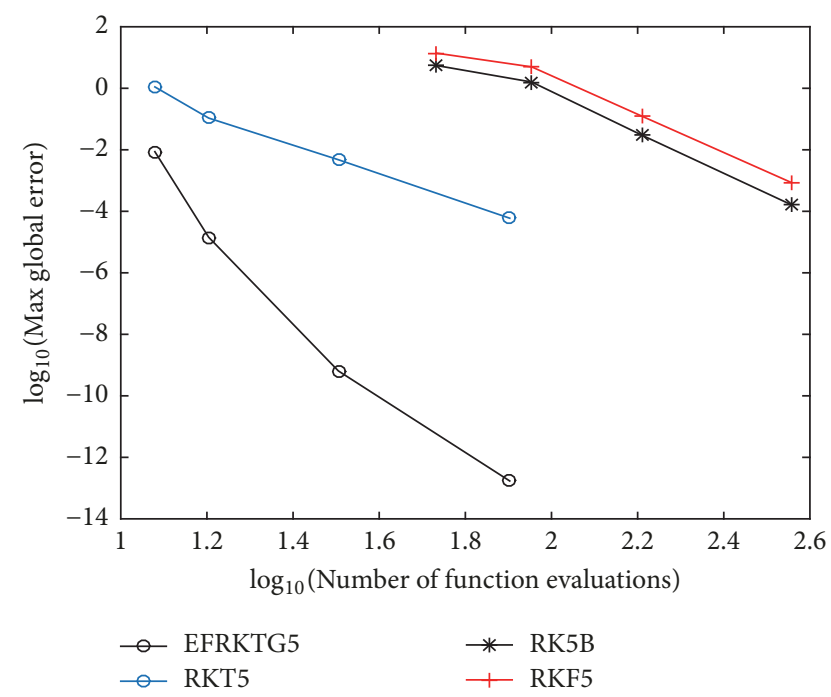

FIGURE 2: The efficiency curve for EFMRKT5, RKT5, RK5B, and RKF5 for Problem 3 with $x_{\text {end }}=2$ and $h=0.1,0.25,0.5,0.75$.

$$
\begin{aligned}
& -\frac{1}{3600} y_{x x} F x y-\frac{1}{144} F y F-\frac{1}{3600} y_{x} F y x x \\
& -\frac{1}{3600} F x z z y_{x x}{ }^{2}+\frac{527}{162000} F x z F \\
& -\frac{1}{10800} y_{x x}{ }^{3} F z z z+\frac{971}{907200} F z F x \\
& +\frac{971}{907200} F z^{2} y_{x x}-\frac{1}{3600} y_{x}^{2} F x y y \\
& +\frac{1}{10800} w^{2} F z y_{x x}-\frac{1}{3600} F z x x y_{x x} \\
& \left.+\frac{1}{10800} w^{2} F y y_{x}\right) h^{6}+O\left(h^{7}\right)
\end{aligned}
$$


TABLE 4: Numerical results for problem in thin film flow (58) taking $h=0.1$ and $k=3$.

\begin{tabular}{lcccccc}
\hline$x$ & RK5B & RKF5 & RKT5 & EMFRKT5 & TFRK & TFMRKT5 \\
\hline 0.0 & 1.000000000 & 1.000000000 & 1.000000000 & 1.000000000 & 1.000000000 & 1.000000000 \\
0.2 & 1.2211551491 & 1.2211551546 & 1.2211551394 & 1.2211551412 & 1.2211551831 & 1.2211551412 \\
0.4 & 1.4881052974 & 1.4881053065 & 1.4881052807 & 1.4881052926 & 1.4881053519 & 1.4881052926 \\
0.6 & 1.8042625677 & 1.8042625794 & 1.8042625459 & 1.8042625786 & 1.8042626364 & 1.8042625786 \\
0.8 & 2.1715228242 & 2.1715228376 & 2.1715227987 & 2.1715228633 & 2.1715229031 & 2.1715228633 \\
1.0 & 2.5909582923 & 2.5909583063 & 2.5909582638 & 2.5909583715 & 2.5909583783 & 2.5909583715 \\
\hline
\end{tabular}

TABLE 5: Numerical results for problem in thin film flow (58) taking $h=0.01$ and $k=3$.

\begin{tabular}{lcccccc}
\hline$x$ & RK5B & RKF5 & RKT5 & EMFRKT5 & TFRK & TFMRKT5 \\
\hline 0.0 & 1.000000000 & 1.000000000 & 1.000000000 & 1.000000000 & 1.000000000 & 1.000000000 \\
0.2 & 1.2211551424 & 1.2211551424 & 1.2211551424 & 1.2211551424 & 1.2211551424 & 1.2211551424 \\
0.4 & 1.4881052842 & 1.4881052842 & 1.4881052842 & 1.4881052842 & 1.4881052842 & 1.4881052842 \\
0.6 & 1.8042625481 & 1.8042625481 & 1.8042625481 & 1.8042625482 & 1.8042625481 & 1.8042625482 \\
0.8 & 2.1715227981 & 2.1715227981 & 2.1715227981 & 2.1715227982 & 2.1715227981 & 2.1715227982 \\
1.0 & 2.5909582591 & 2.5909582591 & 2.5909582591 & 2.5909582592 & 2.5909582591 & 2.5909582592 \\
\hline
\end{tabular}

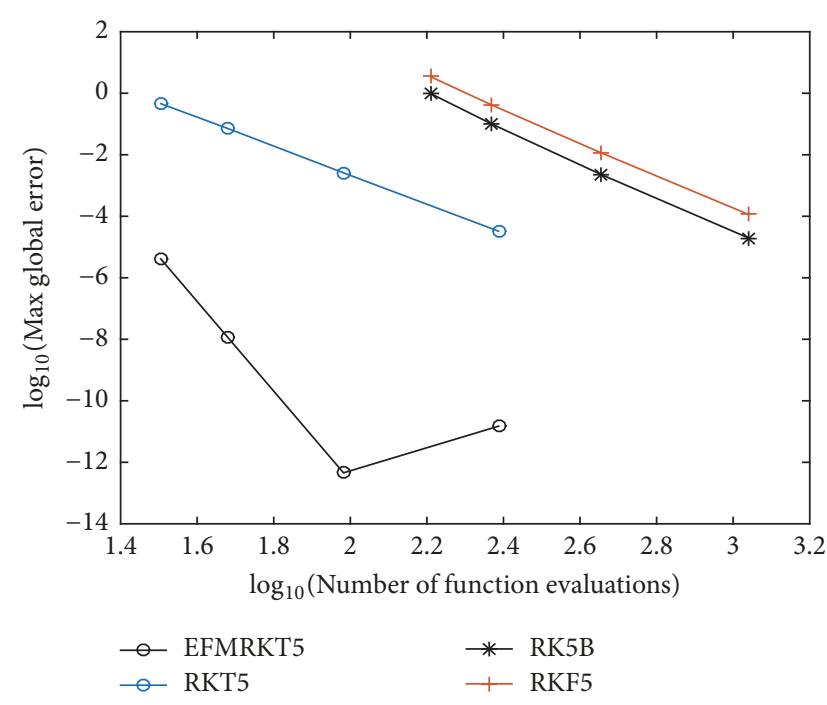

FIgURE 3: The efficiency curve for EFMRKT5, RKT5, RK5B, and RKF5 for Problem 4 with $x_{\text {end }}=6$ and $h=0.1,0.25,0.5,0.75$.

$$
\begin{aligned}
\tau_{n+1}^{\prime} & =\left(\frac{1}{21600} F x x x x+\frac{1811}{756000} F z y_{x} F x y\right. \\
& +\frac{1063}{162000} y_{x x}{ }^{2} F z z z F+\frac{971}{226800} F y y_{x} F x z \\
& +\frac{24853}{4536000} F z y_{x x}{ }^{2} F z z+\frac{15143}{2268000} F z y_{x x} F x z \\
& +\frac{1}{3600} y_{x}^{2} y_{x x}{ }^{2} F z z y y+\frac{1811}{1512000} F z y_{x}^{2} F y y \\
+ & \frac{971}{226800} F z z y_{x x} F x+\frac{971}{226800} y_{x} F y z F x \\
& -\frac{2087}{162000} y_{x x} F y z F+\frac{1}{1800} y_{x} y_{x x} F x x y z
\end{aligned}
$$

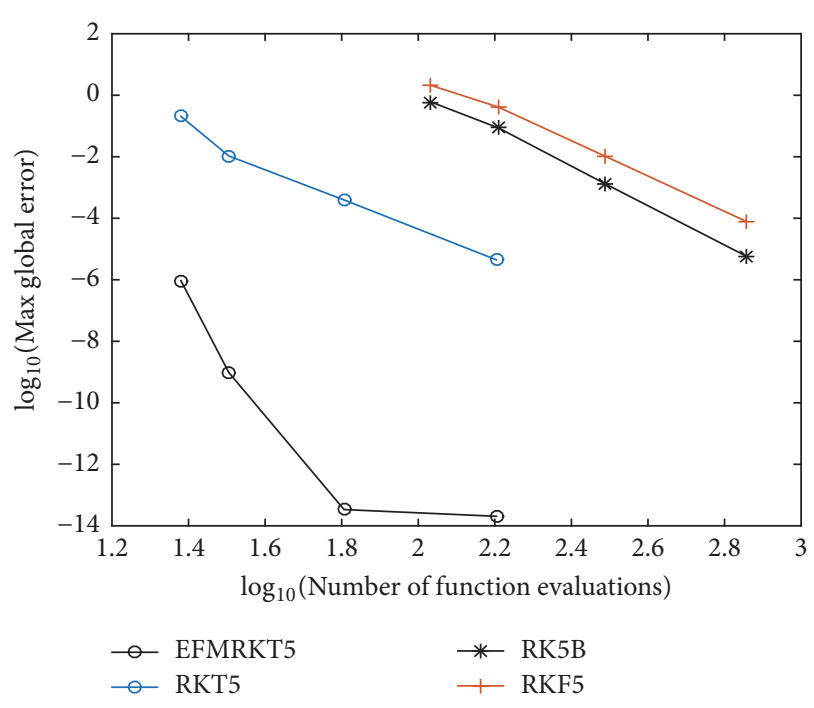

FIGURE 4: The efficiency curve for EFMRKT5, RKT5, RK5B, and RKF5 Problem 5 with $x_{\text {end }}=4$ and $h=0.1,0.25,0.5,0.75$.

$$
\begin{aligned}
& +\frac{1}{1800} y_{x} y_{x x}{ }^{2} F x y z z+\frac{1}{1800} y_{x}^{2} y_{x x} F x y y z \\
& +\frac{1}{5400} y_{x}^{3} F z y y y y_{x x}+\frac{1}{5400} y_{x} y_{x x}{ }^{3} F z z z y \\
& +\frac{971}{226800} F y y_{x}^{2} F y z+\frac{1063}{81000} y_{x} F x y z F \\
& +\frac{1063}{162000} y_{x}^{2} F z y y F+\frac{1}{3600} y_{x}{ }^{2} F y y y y_{x x} \\
& -\frac{8689}{1512000} F z F y y_{x x}+\frac{1}{1800} y_{x} F x y y y_{x x} \\
& +\frac{1063}{81000} F x z z y_{x x} F+\frac{317}{22680000} F z F w^{2}
\end{aligned}
$$


TABLE 6: Comparison of error for problem in thin film flow (58) taking $h=0.1$ and $k=2$.

\begin{tabular}{lccccrr}
\hline$x$ & RK5B & RKF5 & RKT5 & EFMRKT5 & TFRK & TFMRKT5 \\
\hline 0.0 & $0.0000(0)$ & $0.0000(0)$ & $0.0000(0)$ & $0.0000(0)$ & $0.0000(0)$ & $0.0000(0)$ \\
0.2 & $1.0230(-6)$ & $1.0200(-6)$ & $1.2600(-6)$ & $1.0250(-6)$ & $1.0080(-6)$ & $1.0250(-6)$ \\
0.4 & $1.0800(-7)$ & $1.0300(-7)$ & $1.1300(-7)$ & $1.0500(-7)$ & $8.4100(-7)$ & $1.0500(-7)$ \\
0.6 & $2.0000(-9)$ & $7.0000(-9)$ & $5.0000(-8)$ & $2.0000(-8)$ & $3.2000(-8)$ & $2.0000(-8)$ \\
0.8 & $1.2000(-8)$ & $1.7000(-8)$ & $3.0000(-9)$ & $5.300(-8)$ & $4.5000(-8)$ & $5.3000(-8)$ \\
1.0 & $9.3800(-7)$ & $9.3400(-7)$ & $9.4800(-7)$ & $8.6300(-7)$ & $9.0400(-7)$ & $8.6300(-7)$ \\
\hline
\end{tabular}

TABLE 7: Comparison of error for problem in thin film flow (58) taking $h=0.01$ and $k=2$.

\begin{tabular}{lccccrr}
\hline$x$ & RK5B & RKF5 & RKT5 & EFMRKT5 & TFRK & TFMRKT5 \\
\hline 0.0 & $0.0000(0)$ & $0.0000(0)$ & $0.0000(0)$ & $0.0000(0)$ & $0.0000(0)$ & $0.0000(0)$ \\
0.2 & $1.0260(-6)$ & $1.0260(-6)$ & $1.0260(-6)$ & $1.0260(-6)$ & $1.0260(-6)$ & $1.0260(-6)$ \\
0.4 & $6.0000(-7)$ & $6.0000(-7)$ & $6.0000(-7)$ & $6.0000(-7)$ & $6.0000(-7)$ & $6.0000(-7)$ \\
0.6 & $9.0000(-9)$ & $9.0000(-9)$ & $9.0000(-9)$ & $9.0000(-9)$ & $9.0000(-9)$ & $9.0000(-9)$ \\
0.8 & $0.0000(0)$ & $0.0000(0)$ & $0.0000(0)$ & $0.0000(0)$ & $0.0000(0)$ & $0.0000(0)$ \\
1.0 & $9.5400(-7)$ & $9.5400(-7)$ & $9.5400(-7)$ & $9.5400(-7)$ & $9.5400(-7)$ & $9.5400(-7)$ \\
\hline
\end{tabular}

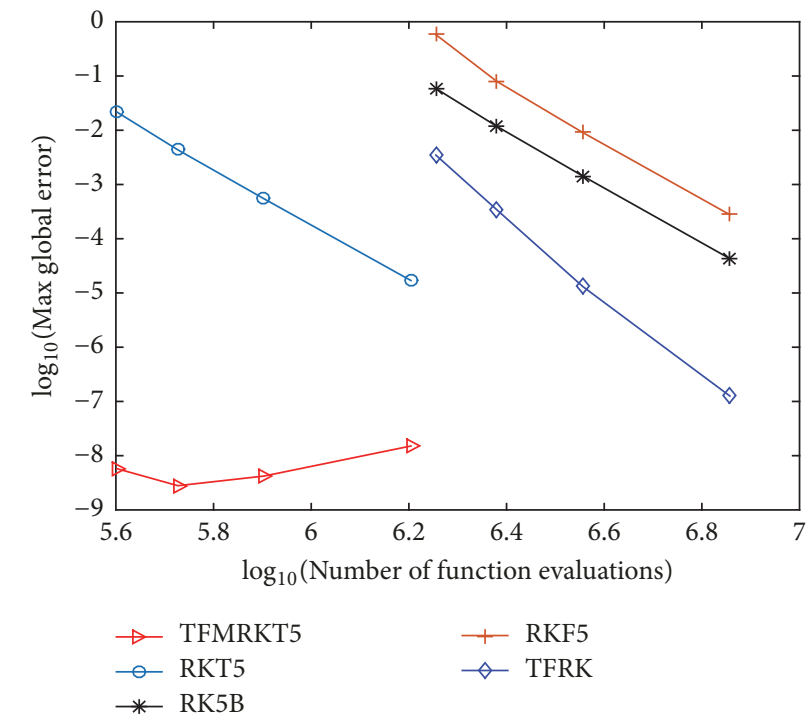

FIGURE 5: The efficiency curve for TFMRKT5, RKT5, RK5B, RKF5, and TFRK for Problem 6 with $x_{\text {end }}=10000$ and $h=$ $0.025,0.05,0.075,0.1$.

$$
\begin{aligned}
& +\frac{1}{1800} y_{x} F z y y y_{x x}{ }^{2}-\frac{7}{360} y_{x} F y y F \\
& +\frac{1}{5400} y_{x} F x y x x+\frac{1}{21600} y_{x}^{4} F y y y y \\
& +\frac{1}{3600} y_{x x} F y x x+\frac{31211}{22680000} F z^{2} F \\
& +\frac{1063}{162000} F z x x F-\frac{7}{360} F F x y+\frac{1}{5400} y_{x}^{3} F x y y y \\
& -\frac{1}{144} F y F x+\frac{1}{5400} y_{x x}{ }^{3} F x z z z \\
& +\frac{1}{21600} y_{x x}{ }^{4} F z z z z+\frac{971}{226800} F x F x z
\end{aligned}
$$

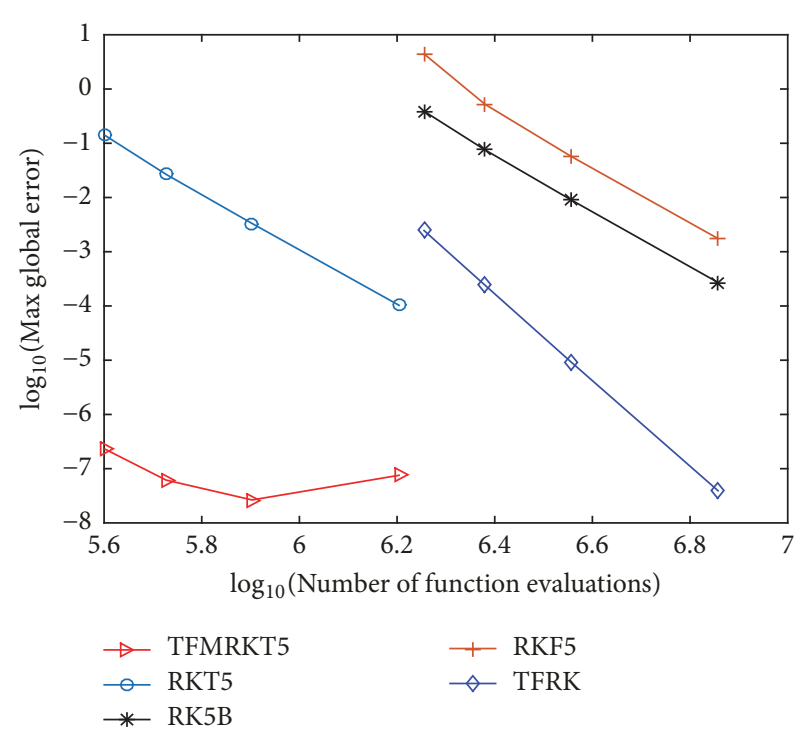

FIGURE 6: The efficiency curve for TFMRKT5, RKT5, RK5B, RKF5, and TFRK for Problem 7 with $x_{\text {end }}=10000$ and $h=$ $0.025,0.05,0.075,0.1$.

$$
\begin{aligned}
& +\frac{1811}{1512000} F z F x x+\frac{1}{3600} y_{x x}{ }^{2} F x x z z \\
& +\frac{1}{3600} y_{x}^{2} F x y x y+\frac{1}{7200} y_{x x}{ }^{2} F y y \\
& +\frac{57889}{14580000} F z z F^{2}+\frac{1}{1800} y_{x x}{ }^{2} F x y z \\
& +\frac{1}{3600} y_{x x}^{3} F z y z-\frac{1}{144} F y^{2} y_{x}+\frac{1}{5400} F x x x z y_{x x} \\
& -\frac{1}{21600} w^{4} F+\frac{1063}{81000} y_{x} y_{x x} F z y z F
\end{aligned}
$$




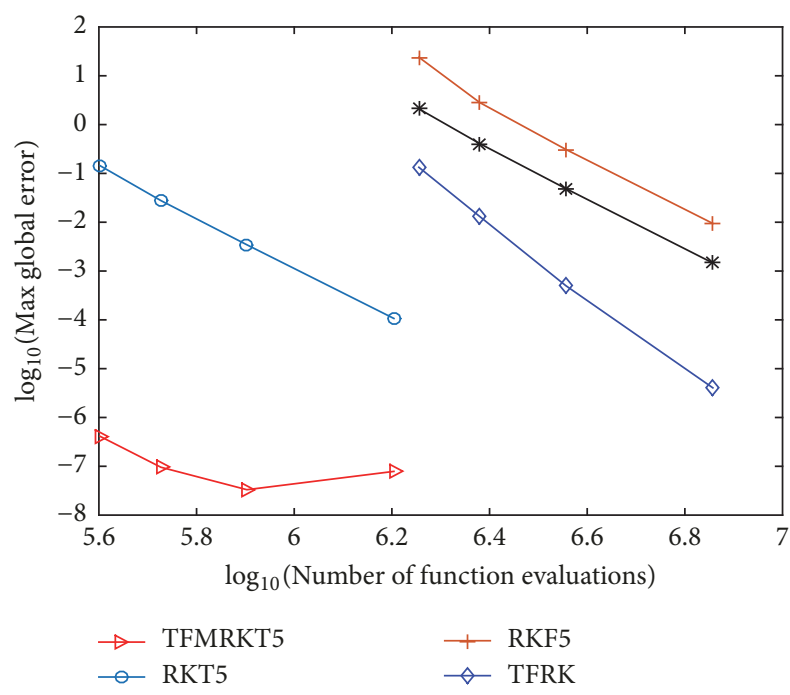

Figure 7: The efficiency curve for TFMRKT5, RKT5, RK5B, RKF5, and TFRK for Problem 8 with $x_{\text {end }}=10000$ and $h=$ $0.025,0.05,0.075,0.1$.

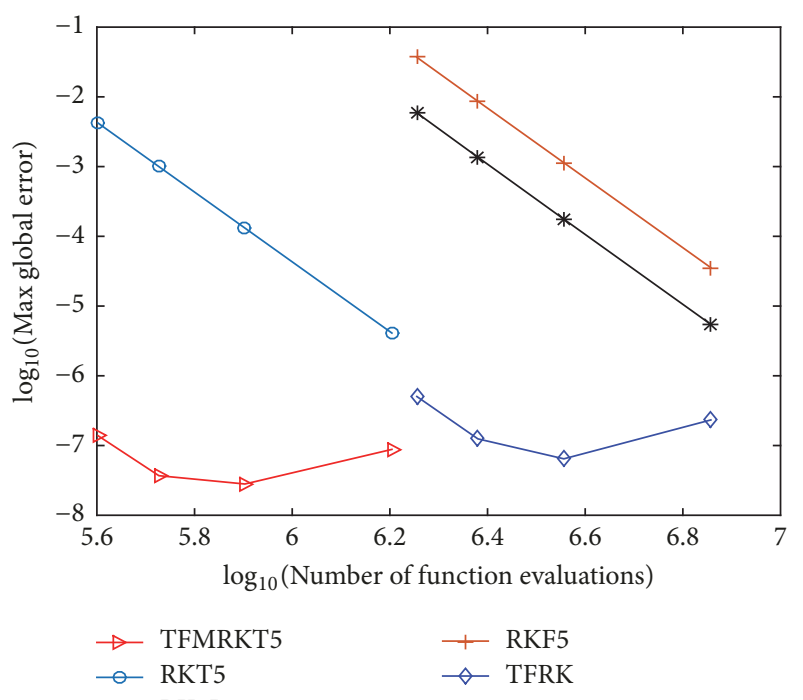

FIGURE 8: The efficiency curve for TFMRKT5, RKT5, RK5B, RKF5, and TFRK for Problem 9 with $x_{\text {end }}=10000$ and $h=$ $0.025,0.05,0.075,0.1$.

$$
\begin{aligned}
&+\left.\frac{971}{226800} F y y_{x} F z z y_{x x}+\frac{15143}{2268000} F z y_{x} F y z y_{x x}\right) \\
& \cdot h^{6}+O\left(h^{7}\right) \\
& \tau_{n+1}^{\prime \prime}=\left(-\frac{7589}{907200} F y y_{x x} F x z-\frac{13777}{907200} y_{x x} F y z F x\right. \\
&+\frac{5011}{302400} y_{x} F z z y_{x x}{ }^{2} F y z+\frac{1193}{40500} y_{x x} F x x z z F
\end{aligned}
$$

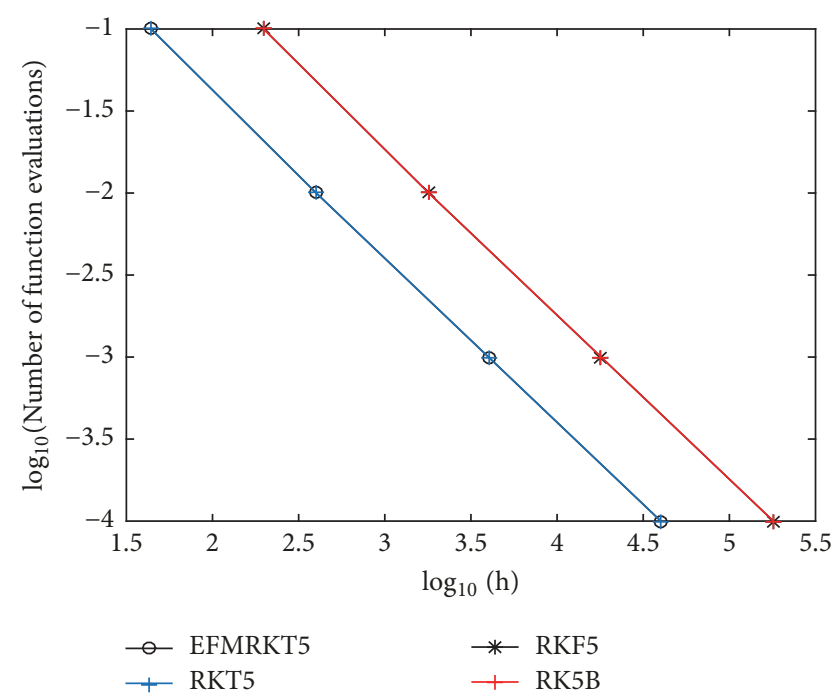

FIGURE 9: Plot of graph for function evaluations against step size $h$ for Problem (58) taking $x_{\text {end }}=1, h=0.1,0.01,0.001,0.0001$, and $k=2$.

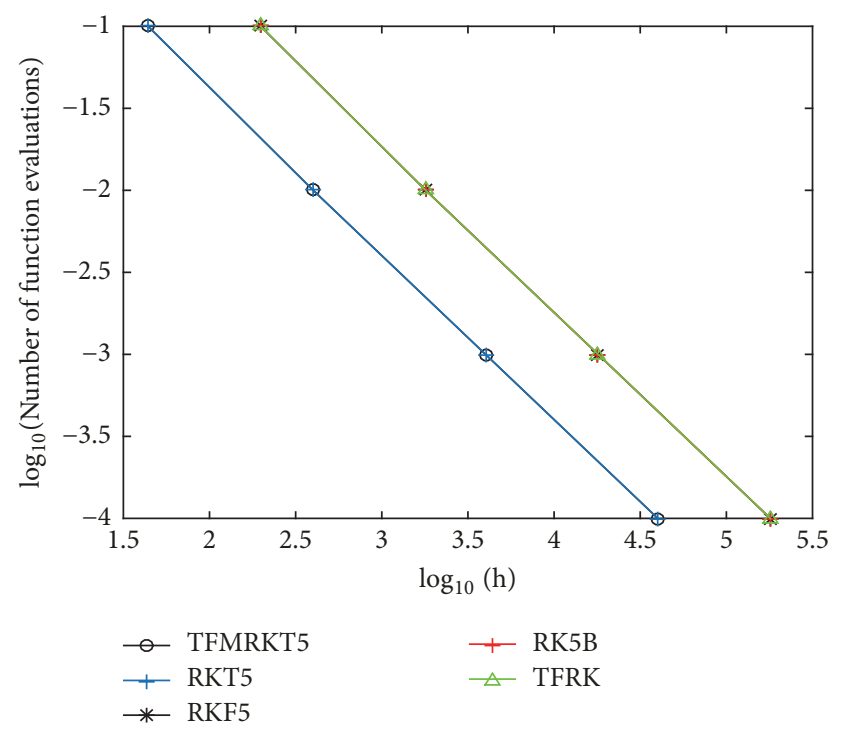

FIGURE 10: Plot of graph for function evaluations against step size $h$ for Problem (58) taking $x_{\text {end }}=1, h=0.1,0.01,0.001,0.0001$ and $k=2$.

$$
\begin{aligned}
& +\frac{32703767}{3061800000} F z F F x z+\frac{3551}{3189375} F z y_{x}^{3} F y y y \\
& -\frac{1}{5400} y_{x}^{3} F x y y y z y_{x x}-\frac{1}{10800} y_{x x}{ }^{2} F x x x z z \\
& +\frac{276941}{14580000} y_{x x} F z z z F^{2}+\frac{10163}{907200} y_{x}^{2} F z y y F x \\
& +\frac{10163}{453600} F x F x z z y_{x x}-\frac{1}{1800} y_{x} y_{x x}{ }^{2} F x y y z \\
& -\frac{1}{3600} y_{x} y_{x x}{ }^{3} F z z y y+\frac{1511}{7560000} F w^{2} F y z y_{x}
\end{aligned}
$$




$$
\begin{aligned}
& -\frac{19}{720} y_{x} F y y F x-\frac{392059}{17010000} F z y_{x} F y y y_{x x} \\
& +\frac{5011}{907200} y_{x}^{2} F y y F z z y_{x x} \\
& +\frac{989489}{68040000} F z y_{x}^{2} F z y y y_{x x} \\
& +\frac{5011}{226800} y_{x} F x z F y z y_{x x}+\frac{10163}{453600} F y y_{x} F x z z y_{x x} \\
& +\frac{5011}{453600} y_{x} F x y F z z y_{x x} \\
& +\frac{989489}{34020000} F z y_{x} F x y z y_{x x} \\
& +\frac{10163}{453600} y_{x} F x F z y z y_{x x} \\
& +\frac{10163}{453600} F y y_{x}^{2} F z y z y_{x x} \\
& +\frac{875857}{34020000} y_{x} y_{x x}{ }^{2} \mathrm{~F} z y z \mathrm{Fz} \\
& -\frac{1}{10800} y_{x}{ }^{2} y_{x x}{ }^{3} F z z z y y-\frac{1}{21600} y_{x} y_{x x}{ }^{4} F z z z z y \\
& -\frac{1}{3600} y_{x}^{2} F x x y y z y_{x x}+\frac{3551}{1063125} F z y_{x}^{2} F x y y \\
& +\frac{2513939}{204120000} y_{x x}^{3} \mathrm{~F} z z z \mathrm{Fz} \\
& -\frac{392059}{17010000} F z y_{x x} F x y+\frac{5011}{302400} F x z y_{x x}{ }^{2} F z z \\
& +\frac{5011}{907200} y_{x} F y z F x x+\frac{875857}{34020000} F z y_{x x}{ }^{2} F x z z \\
& +\frac{10163}{907200} F y y_{x} y_{x x}{ }^{2} F z z z+\frac{1193}{121500} y_{x}^{3} F z y y y F \\
& -\frac{1}{3600} y_{x}{ }^{2} y_{x x}{ }^{2} F x y y z z+\frac{1193}{40500} y_{x} F F x x y z \\
& -\frac{1}{10800} y_{x}^{3} F y y y y y_{x x}+\frac{1193}{40500} y_{x}^{2} F F x y y z \\
& +\frac{4169}{34020000} F z w^{2} F x-\frac{1}{10800} y_{x}^{2} F x x x y y \\
& +\frac{5011}{907200} F z z^{2} y_{x x}{ }^{3}-\frac{1}{21600} y_{x}^{4} F z y y y y y_{x x} \\
& +\frac{5011}{453600} y_{x}{ }^{2} F y z^{2} y_{x x}-\frac{433}{10125} y_{x x} F x y z F \\
& -\frac{1}{10800} y_{x}^{3} F x x y y y+\frac{5011}{907200} F x z F x x \\
& -\frac{1}{108000} y_{x x}{ }^{5} F z z z z z+\frac{1511}{7560000} F w^{2} F x z \\
& -\frac{13}{360} y_{x x} F y y F-\frac{1}{3600} y_{x} F x y x y y_{x x}
\end{aligned}
$$$$
-\frac{1}{3600} y_{x x}{ }^{2} \mathrm{~F} x x y z-\frac{1}{3600} y_{x x}{ }^{3} \mathrm{~F} x y z z
$$$$
-\frac{179}{567000} F z F y F-\frac{1}{21600} y_{x}^{4} F x y y y y
$$$$
-\frac{1}{5400} y_{x} F x x x y z y_{x x}+\frac{989489}{68040000} F z x x F z y_{x x}
$$$$
+\frac{21449}{1632960} F z z F F x+\frac{46577}{34992000} F z^{2} F y y_{x}
$$$$
-\frac{1}{3600} y_{x}{ }^{2} F x y y y y_{x x}+\frac{3551}{1063125} F z y_{x} F y x x
$$$$
+\frac{10163}{907200} F y y_{x} F z x x+\frac{276941}{14580000} y_{x} F^{2} F z y z
$$$$
-\frac{1289}{907200} F y y_{x x}{ }^{2} F z z-\frac{1}{10800} y_{x x}{ }^{3} F x x z z z
$$$$
+\frac{10163}{453600} F y y_{x}^{2} F x y z+\frac{1}{108000} w^{4} F z y_{x x}
$$$$
+\frac{5011}{453600} y_{x} F x z F x y+\frac{10163}{453600} y_{x} F x F x y z
$$$$
+\frac{1193}{40500} y_{x x}{ }^{2} \mathrm{~F} x z z z F+\frac{1193}{121500} y_{x x}{ }^{3} \mathrm{~F} z z z z \mathrm{~F}
$$$$
-\frac{1187}{50400} F y y_{x} F y z y_{x x}+\frac{10163}{907200} F x F z x x
$$$$
-\frac{1}{21600} y_{x} F x x x x y+\frac{276941}{14580000} F x z z F^{2}
$$$$
-\frac{1}{7200} y_{x x}{ }^{2} F x y y-\frac{806011}{68040000} F z y_{x x}{ }^{2} F y z
$$$$
-\frac{13}{360} y_{x}^{2} F y y y F-\frac{13}{180} y_{x} F x y y F
$$$$
-\frac{1}{7200} y_{x} F y y y y_{x x}{ }^{2}-\frac{1}{5400} y_{x} y_{x x}{ }^{3} F x y z z z
$$$$
+\frac{32703767}{3061800000} F z y_{x} F y z F
$$$$
+\frac{4169}{34020000} F z w^{2} F y y_{x}+\frac{21449}{1632960} F y y_{x} F z z F
$$$$
+\frac{1}{108000} w^{4} F x+\frac{5011}{907200} y_{x}^{3} F y y F y z
$$$$
-\frac{1}{10800} y_{x}{ }^{3} y_{x x}{ }^{2} F z z y y y-\frac{29}{720} F y y_{x} F x y
$$$$
-\frac{539}{81000} y_{x x}{ }^{2} F z y z F-\frac{19}{720} F x F x y
$$$$
-\frac{1}{21600} y_{x x}{ }^{4} F x z z z z-\frac{433}{10125} y_{x} F z y y y_{x x} F
$$$$
-\frac{1}{108000} F x x x x x+\frac{1193}{40500} y_{x} y_{x x}{ }^{2} F z z z y F
$$$$
-\frac{1}{144} F y F x x+\frac{1193}{40500} y_{x}^{2} y_{x x} F z z y y F
$$ 


$$
\begin{aligned}
& +\frac{1193}{20250} y_{x} y_{x x} F x y z z F+\frac{1511}{7560000} F w^{2} F z z y_{x x} \\
& +\frac{36460321}{1530900000} F z y_{x x} F z z F+\frac{5011}{907200} F z z y_{x x} F x x \\
& +\frac{46577}{34992000} F z^{2} F x+\frac{46577}{34992000} F z^{3} y_{x x} \\
& -\frac{1}{10800} F^{2} F y z-\frac{1}{3600} y_{x} y_{x x}{ }^{2} F x x y z z \\
& +\frac{5011}{453600} y_{x}^{2} F y z F x y-\frac{1}{10800} y_{x x} F x y x x \\
& -\frac{13}{360} F F y x x-\frac{1}{108000} y_{x}^{5} F y y y y y \\
& -\frac{1}{144} F y^{2} y_{x x}+\frac{1193}{121500} F x x x z F \\
& -\frac{1}{21600} F x x x x z y_{x x}-\frac{1}{7200} y_{x x}{ }^{3} F z y y \\
& +\frac{1}{108000} w^{4} F y y_{x}+\frac{10163}{907200} F y y_{x}^{3} F z y y \\
& -\frac{1}{30} y_{x}^{2} F y y F y-\frac{1}{10800} y_{x x}{ }^{4} F z z z y \\
& +\frac{4169}{34020000} F z^{2} w^{2} y_{x x}+\frac{5011}{453600} F x z^{2} y_{x x} \\
& -\frac{1}{3600} y_{x}{ }^{2} F z y y y y_{x x}{ }^{2}+\frac{5011}{907200} y_{x}{ }^{2} F y y F x z \\
& \left.+\frac{10163}{907200} y_{x x}{ }^{2} F z z z F x+\frac{3551}{3189375} F z F x x x\right) h^{6} \\
& +O\left(h^{7}\right)
\end{aligned}
$$

The principal local truncation errors for $y, y^{\prime}$, and $y^{\prime \prime}$ (i.e., $\left.\tau_{n+1}, \tau_{n+1}^{\prime}, \tau_{n+1}^{\prime \prime}\right)$ for TFMRKT5 are as follows:

$$
\begin{aligned}
\tau_{n+1} & =\left(-\frac{1}{10800} y_{x}^{3} F y y y-\frac{1}{3600} F z x x y_{x x}\right. \\
& -\frac{1}{3600} y_{x} F y y y_{x x}-\frac{1}{3600} y_{x x}{ }^{2} F y z \\
& +\frac{527}{162000} F z z y_{x x} F-\frac{1}{1800} y_{x} F x y z y_{x x} \\
& -\frac{1}{3600} y_{x}^{2} F z y y y_{x x}+\frac{527}{162000} y_{x} F y z F \\
& +\frac{971}{907200} F z F y y_{x}-\frac{1}{3600} F x z z y_{x x}{ }^{2} \\
& -\frac{1}{3600} y_{x}^{2} F x y y-\frac{1}{10800} y_{x x}{ }^{3} F z z z \\
& +\frac{971}{907200} F z F x+\frac{971}{907200} F z^{2} y_{x x} \\
& +\frac{527}{162000} F x z F-\frac{1}{144} F y F-\frac{1}{3600} y_{x} y_{x x}{ }^{2} F z y z
\end{aligned}
$$

$$
\begin{aligned}
& -\frac{1}{3600} y_{x x} F x y-\frac{1}{10800} w^{2} F z y_{x x}-\frac{1}{10800} F x x x \\
& -\frac{1}{3600} y_{x} F y x x-\frac{1}{10800} w^{2} F y y_{x} \\
& \left.-\frac{1}{10800} w^{2} F x\right) h^{6}+O\left(h^{7}\right)
\end{aligned}
$$$$
\tau_{n+1}^{\prime}=\left(-\frac{2087}{162000} y_{x x} F y z F+\frac{1063}{162000} y_{x}^{2} F z y y F\right.
$$$$
-\frac{8689}{1512000} F z F y y_{x x}+\frac{971}{226800} F y y_{x} F x z
$$$$
+\frac{1811}{756000} F z y_{x} F x y+\frac{1}{1800} y_{x}^{2} y_{x x} F x y y z
$$$$
+\frac{1}{3600} y_{x}^{2} y_{x x}^{2} F z z y y+\frac{1}{1800} y_{x} y_{x x} F x x y z
$$$$
+\frac{1}{1800} y_{x} y_{x x}^{2} F x y z z+\frac{15143}{2268000} F z y_{x x} F x z
$$$$
+\frac{24853}{4536000} F z y_{x x}{ }^{2} F z z+\frac{971}{226800} y_{x} F y z F x
$$$$
+\frac{971}{226800} F z z y_{x x} F x+\frac{1}{5400} y_{x} y_{x x}^{3} F z z z y
$$$$
+\frac{1}{5400} y_{x}^{3} F z y y y y_{x x}+\frac{1}{1800} y_{x} F z y y y_{x x}{ }^{2}
$$$$
+\frac{971}{226800} F y y_{x}^{2} F y z+\frac{1811}{1512000} F z y_{x}^{2} F y y
$$$$
-\frac{317}{22680000} F z F w^{2}+\frac{1}{3600} y_{x}^{2} F y y y y_{x x}
$$$$
+\frac{1063}{81000} F x z z y_{x x} F+\frac{1063}{81000} y_{x} F x y z F
$$$$
+\frac{1}{1800} y_{x} F x y y y_{x x}+\frac{1063}{162000} y_{x x}{ }^{2} F z z z F
$$$$
-\frac{7}{360} y_{x} F y y F+\frac{1063}{81000} y_{x} y_{x x} F z y z F
$$$$
+\frac{971}{226800} F y y_{x} F z z y_{x x}+\frac{15143}{2268000} F z y_{x} F y z y_{x x}
$$$$
+\frac{1}{21600} F x x x x-\frac{1}{21600} w^{4} F+\frac{1}{5400} F x x x z y_{x x}
$$$$
+\frac{1}{3600} y_{x x} F y x x+\frac{1}{1800} y_{x x}{ }^{2} F x y z
$$$$
+\frac{1}{7200} y_{x x}^{2} F y y+\frac{57889}{14580000} F z z F^{2}
$$$$
+\frac{1}{3600} y_{x x}{ }^{3} F z y z+\frac{1}{21600} y_{x}{ }^{4} F y y y y
$$$$
+\frac{1}{5400} y_{x x}{ }^{3} \mathrm{~F} x z z z+\frac{1}{21600} y_{x x}{ }^{4} \mathrm{~F} z z z z
$$$$
+\frac{1}{5400} y_{x}^{3} F x y y y-\frac{1}{144} F y F x+\frac{1}{5400} y_{x} F x y x x
$$ 


$$
\begin{aligned}
& +\frac{1}{3600} y_{x x}{ }^{2} F x x z z+\frac{1}{3600} y_{x}{ }^{2} F x y x y \\
& +\frac{971}{226800} F x z F x+\frac{1811}{1512000} F z F x x-\frac{1}{144} F y^{2} y_{x} \\
& +\frac{31211}{22680000} F z^{2} F+\frac{1063}{162000} F z x x F \\
& \left.-\frac{7}{360} F F x y\right) h^{6}+O\left(h^{7}\right) \\
& \tau_{n+1}^{\prime \prime}=\left(\frac{10373}{453600} F y y_{x} y_{x x} F x z z\right. \\
& +\frac{10373}{907200} F y y_{x} y_{x x}{ }^{2} F z z z+\frac{1717}{75600} y_{x} F y z y_{x x} F x z \\
& +\frac{10373}{453600} y_{x} y_{x x} F z y z F x \\
& +\frac{298369}{11340000} y_{x} y_{x x}^{2} \mathrm{~F} z y z \mathrm{Fz} \\
& +\frac{1717}{151200} y_{x} F z z y_{x x} F x y \\
& +\frac{1717}{100800} y_{x} F z z y_{x x}{ }^{2} F y z+\frac{7811}{5832000} F z^{2} F y y_{x} \\
& -\frac{27487}{648000} y_{x x} F x y z F-\frac{67}{7560000} F z w^{2} F x \\
& -\frac{67}{7560000} F z^{2} w^{2} y_{x x}-\frac{13}{360} y_{x}^{2} F y y y F \\
& -\frac{13}{180} y_{x} F x y y F-\frac{13}{360} y_{x x} F y y F \\
& -\frac{4087}{648000} y_{x x}{ }^{2} F z y z F-\frac{61}{378000} F z F y F \\
& -\frac{2483}{302400} F y y_{x x} F x z-\frac{130103}{5670000} F z y_{x x} F x y \\
& +\frac{19313}{648000} y_{x}^{2} F F x y y z+\frac{1717}{302400} y_{x}^{3} F y y F y z \\
& +\frac{9761}{8505000} F z y_{x}^{3} F y y y-\frac{1}{30} F y y_{x}^{2} F y y \\
& +\frac{10373}{907200} y_{x}{ }^{3} F z y y F y-\frac{1}{3600} y_{x}{ }^{2} F x y y y y_{x x} \\
& +\frac{19313}{648000} y_{x} F F x x y z+\frac{10959589}{1020600000} F z F F x z \\
& -\frac{13567}{907200} y_{x x} F y z F x+\frac{337511}{25515000} F z z F F x \\
& +\frac{19313}{1944000} y_{x}{ }^{3} F z y y y F-\frac{1}{10800} y_{x}^{3} F y y y y y_{x x} \\
& +\frac{19313}{648000} y_{x x}{ }^{2} F x z z z F+\frac{19313}{1944000} y_{x x}{ }^{3} F z z z z F \\
& +\frac{19313}{648000} y_{x x} F x x z z F-\frac{1}{3600} y_{x} F x y x y y_{x x}
\end{aligned}
$$

$$
\begin{aligned}
& +\frac{1717}{302400} y_{x}^{2} F y y F x z+\frac{1}{108000} w^{4} F z y_{x x} \\
& +\frac{1}{108000} w^{4} F y y_{x}+\frac{9761}{2835000} F z y_{x}^{2} F x y y \\
& -\frac{29}{720} F y y_{x} F x y+\frac{10373}{453600} y_{x} F x y z F x \\
& +\frac{10373}{453600} F x z z y_{x x} F x+\frac{298369}{11340000} F x z z y_{x x}{ }^{2} F z \\
& +\frac{10373}{907200} y_{x x}{ }^{2} \mathrm{~F} z z z \mathrm{~F} x+\frac{856063}{68040000} y_{x x}{ }^{3} \mathrm{~F} z z z \mathrm{~F} z \\
& -\frac{19}{720} y_{x} F y y F x+\frac{10373}{907200} y_{x}{ }^{2} F z y y F x \\
& +\frac{1717}{151200} y_{x} F x z F x y+\frac{10373}{907200} F y y_{x} F z x x \\
& +\frac{9761}{2835000} F z y_{x} F y x x-\frac{1}{3600} y_{x}^{2} y_{x x} F x x y y z \\
& -\frac{1}{3600} y_{x}{ }^{2} y_{x x}{ }^{2} F x y y z z-\frac{1}{10800} y_{x}{ }^{2} y_{x x}{ }^{3} F z z z y y \\
& -\frac{1}{5400} y_{x} y_{x x} F x x x y z-\frac{1}{3600} y_{x} y_{x x}{ }^{2} F x x y z z \\
& -\frac{1}{5400} y_{x} y_{x x}{ }^{3} \mathrm{~F} x y z z z+\frac{1717}{100800} F x z y_{x x}{ }^{2} \mathrm{~F} z z \\
& +\frac{112471}{7560000} F z y_{x x} F z x x+\frac{1717}{302400} y_{x} F y z F x x \\
& +\frac{1717}{302400} F z z y_{x x} F x x-\frac{1}{21600} y_{x} y_{x x}^{4} F z z z z y \\
& -\frac{1}{5400} y_{x}^{3} y_{x x} F x y y y z-\frac{1}{10800} y_{x}{ }^{3} y_{x x}{ }^{2} F z z y y y \\
& +\frac{1717}{151200} y_{x}^{2} F y z F x y+\frac{1717}{151200} y_{x}^{2} F y z^{2} y_{x x} \\
& +\frac{10373}{453600} F y y_{x}{ }^{2} F x y z-\frac{1}{21600} y_{x}{ }^{4} F z y y y y y_{x x} \\
& -\frac{1}{7200} y_{x} F y y y y_{x x}^{2}+\frac{1114379}{58320000} y_{x x} F z z z F^{2} \\
& -\frac{1}{1800} y_{x} y_{x x}{ }^{2} \mathrm{~F} x y y z-\frac{1}{3600} y_{x} y_{x x}{ }^{3} \mathrm{~F} z z y y \\
& -\frac{1}{3600} y_{x}{ }^{2} F z y y y y_{x x}{ }^{2}+\frac{1114379}{58320000} y_{x} F^{2} F z y z \\
& -\frac{383}{302400} F y y_{x x}{ }^{2} F z z-\frac{87029}{7560000} F z y_{x x}{ }^{2} F y z \\
& -\frac{1}{108000} F x x x x x+\frac{1}{108000} w^{4} F x \\
& -\frac{1}{108000} y_{x}{ }^{5} F y y y y y+\frac{1114379}{58320000} F x z z F^{2} \\
& -\frac{1}{7200} y_{x x}^{2} F x y y+\frac{11}{43200} F^{2} F y z \\
& -\frac{1}{3600} y_{x x}{ }^{2} \mathrm{~F} x x y z-\frac{1}{3600} y_{x x}{ }^{3} \mathrm{~F} x y z z
\end{aligned}
$$




$$
\begin{aligned}
& -\frac{1}{10800} y_{x x}{ }^{4} F z z z y-\frac{1}{7200} y_{x x}{ }^{3} F z y y \\
& -\frac{13}{360} F F y x x-\frac{1}{21600} F x x x x z y_{x x} \\
& -\frac{1}{10800} y_{x x} F x y x x-\frac{1}{144} F y^{2} y_{x x} \\
& +\frac{1717}{151200} F x z^{2} y_{x x}+\frac{1717}{302400} F z z^{2} y_{x x}{ }^{3} \\
& -\frac{1}{21600} y_{x}^{4} \mathrm{~F} x y y y y-\frac{1}{21600} y_{x x}{ }^{4} \mathrm{~F} x z z z z \\
& -\frac{1}{108000} y_{x x}{ }^{5} F z z z z z-\frac{19}{720} F x F x y \\
& -\frac{1}{144} F y F x x+\frac{1717}{302400} F x x F x z \\
& +\frac{9761}{8505000} F z F x x x+\frac{7811}{5832000} F z^{2} F x \\
& +\frac{7811}{5832000} F z^{3} y_{x x}-\frac{1}{10800} y_{x x}{ }^{3} F x x z z z \\
& -\frac{1}{10800} y_{x}^{3} F x x y y y-\frac{1}{21600} y_{x} F x x x x y \\
& -\frac{1}{10800} y_{x x}{ }^{2} F x x x z z-\frac{1}{10800} y_{x}{ }^{2} F x x x y y \\
& +\frac{10373}{907200} F x F z x x+\frac{19313}{1944000} F x x x z F \\
& -\frac{1331}{45360000} F x z F w^{2}+\frac{112471}{3780000} F z y_{x} y_{x x} F x y z \\
& +\frac{1717}{302400} y_{x}^{2} F y y F z z y_{x x} \\
& +\frac{112471}{7560000} F z y_{x}^{2} F z y y y_{x x} \\
& +\frac{10373}{453600} F y y_{x}{ }^{2} F z y z y_{x x}-\frac{27487}{648000} y_{x} F z y y F y_{x x} \\
& +\frac{19313}{648000} y_{x}^{2} y_{x x} F z z y y F \\
& +\frac{19313}{324000} y_{x} y_{x x} F x y z z F+\frac{905927}{37800000} F z y_{x x} F z z F \\
& +\frac{19313}{648000} y_{x} y_{x x}{ }^{2} F z z z y F+\frac{337511}{25515000} F y y_{x} F z z F \\
& +\frac{10959589}{1020600000} F z y_{x} F y z F-\frac{2627}{113400} F y y_{x} F y z y_{x x} \\
& -\frac{130103}{5670000} F z y_{x} F y y y_{x x}-\frac{1331}{45360000} y_{x x} F z z F w^{2} \\
& \left.-\frac{1331}{45360000} y_{x} F y z F w^{2}-\frac{67}{7560000} F z w^{2} F y y_{x}\right) \\
& \cdot h^{6}+O\left(h^{7}\right)
\end{aligned}
$$

\section{Conflicts of Interest}

The authors declare that there are no conflicts of interest regarding the publication of this paper.

\section{References}

[1] B. Paternoster, "Runge-Kutta(-Nyström) methods for ODEs with periodic solutions based on trigonometric polynomials," Applied Numerical Mathematics, vol. 28, no. 2-4, pp. 401-412, 1998.

[2] G. Vanden Berghe, H. De Meyer, M. Van Daele, and T. Van Hecke, "Exponentially fitted Runge-Kutta methods," Journal of Computational and Applied Mathematics, vol. 125, no. 1-2, pp. 107-115, 2000.

[3] T. E. Simos, "Exponentially fitted Runge-Kutta methods for the numerical solution of the Schrödinger equation and related problems," Computational Materials Science, vol. 18, no. 3-4, pp. 315-332, 2000.

[4] Z. Kalogiratou and T. E. Simos, "Construction of trigonometrically and exponentially fitted Runge-Kutta-Nyström methods for the numerical solution of the schrödinger equation and related problems - a method of 8th algebraic order," Journal of Mathematical Chemistry, vol. 31, no. 2, pp. 211-232, 2002.

[5] Z. Kalogiratou, T. Monovasilis, and T. E. Simos, "Computation of the eigenvalues of the Schrödinger equation by exponentially-fitted Runge-Kutta-Nyström methods," Computer Physics Communications, vol. 180, no. 2, pp. 167-176, 2009.

[6] T. E. Simos, "Exponentially-fitted Runge-Kutta-Nyström method for the numerical solution of initial-value problems with oscillating solutions," Applied Mathematics Letters, vol. 15, no. 2, pp. 217-225, 2002.

[7] D. P. Sakas and T. E. Simos, "A fifth algebraic order trigonometrically-fitted modified Runge-Kutta Zonneveld method for the numerical solution of orbital problems," Mathematical and Computer Modelling, vol. 42, no. 7-8, pp. 903-920, 2005.

[8] H. Van De Vyver, "A Runge-Kutta-Nyström pair for the numerical integration of perturbed oscillators," Computer Physics Communications, vol. 167, no. 2, pp. 129-142, 2005.

[9] H. Yang and X. Wu, "Trigonometrically-fitted ARKN methods for perturbed oscillators," Applied Numerical Mathematics, vol. 58, no. 9, pp. 1375-1395, 2008.

[10] M. A. Demba, N. Senu, and F. Ismail, "Trigonometricallyfitted explicit four-stage fourth-order Runge-Kutta-Nyström method for the solution of initial value problems with oscillatory behavior," The Global Journal of Pure and Applied Mathematics (GJPAM), vol. 12, no. 1, pp. 67-80, 2016.

[11] M. Hanan, "Oscillation criteria for third-order linear differential equations," Pacific Journal of Mathematics, vol. 11, pp. 919944, 1961.

[12] J. Rovder, "Oscillation criteria for third-order linear differential equations," Matematický časopis, vol. 25, no. 3, pp. 231-244, 1975.

[13] A. C. Lazer, "The behavior of solutions of the differential equation $y^{\prime \prime \prime}+p(\mathrm{x}) \mathrm{y}^{\prime}+\mathrm{q}(x) y=0$," Pacific Journal of Mathematics, vol. 17, pp. 435-466, 1966.

[14] G. D. Jones, "Properties of solutions of a class of third-order differential equations," Journal of Mathematical Analysis and Applications, vol. 48, pp. 165-169, 1974.

[15] F. A. Fawzi, N. Senu, F. Ismail, and Z. A. Majid, "An efficient of direct integrator of Runge-Kutta Type Method for Solving $y^{\prime \prime \prime}=f\left(x, y, y^{\prime}\right)$ with Application to Thin Film Flow Problem," 
International Journal of Pure and Applied Mathematics, vol. 117, no. 4 , accepted.

[16] J. C. Butcher, Numerical Methods for Ordinary Differential Equations, John Wiley \& Sons, New York, NY, USA, 2nd edition, 2008.

[17] J. D. Lambert, Numerical Methods for Ordinary Differential Systems. The Initial Value Problem, John Wiley \& Sons, New York, NY, USA, 1993.

[18] Z. A. Anastassi and T. E. Simos, "Trigonometrically fitted Runge-Kutta methods for the numerical solution of the Schrödinger equation," Journal of Mathematical Chemistry, vol. 37, no. 3, pp. 281-293, 2005.

[19] E. Momoniat and F. M. Mahomed, "Symmetry reduction and numerical solution of a third-order ODE from thin film flow," Mathematical \& Computational Applications, vol. 15, no. 4, pp. 709-719, 2010.

[20] E. O. Tuck and L. W. Schwartz, "A numerical and asymptotic study of some third-order ordinary differential equations relevant to draining and coating flows," SIAM Review. A Publication of the Society for Industrial and Applied Mathematics, vol. 32, no. 3, pp. 453-469, 1990.

[21] J. Biazar, E. Babolian, and R. Islam, "Solution of the system of ordinary differential equations by Adomian decomposition method," Applied Mathematics and Computation, vol. 147, no. 3, pp. 713-719, 2004.

[22] M. Mechee, N. Senu, F. Ismail, B. Nikouravan, and Z. Siri, “A three-stage fifth-order Runge-Kutta method for directly solving special third-order differential equation with application to thin film flow problem," Mathematical Problems in Engineering, vol. 2013, Article ID 795397, 7 pages, 2013. 


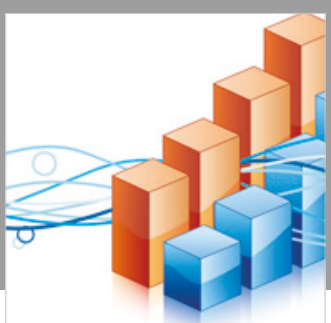

Advances in

Operations Research

\section{-n-m}
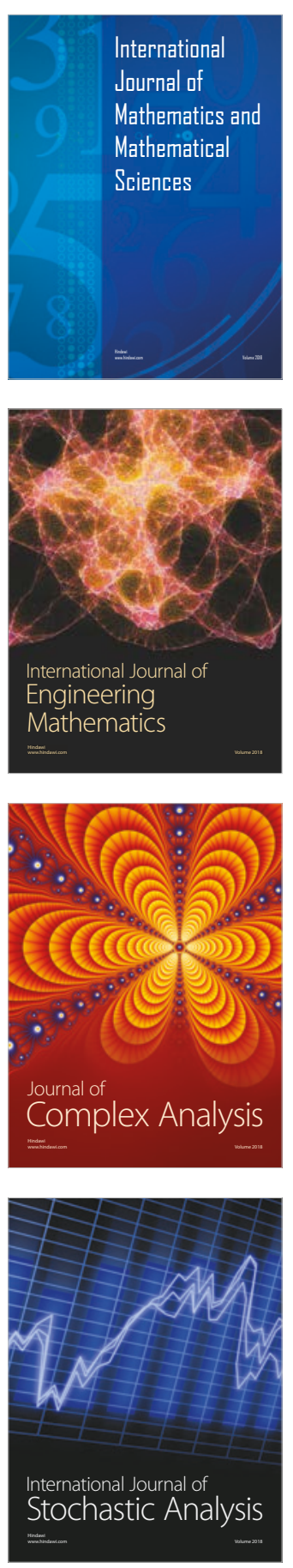
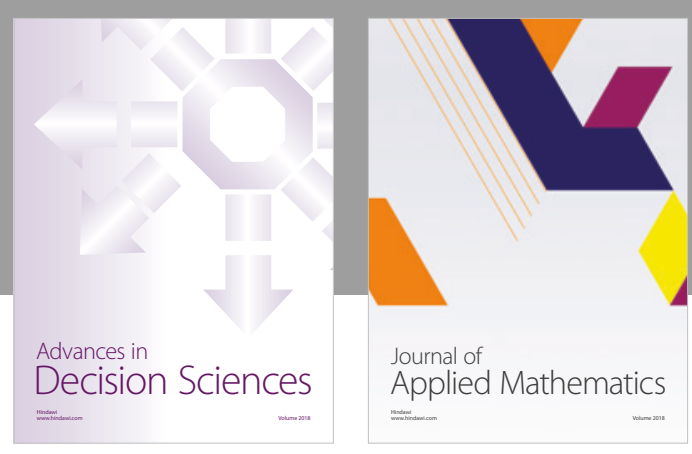

Journal of

Applied Mathematics
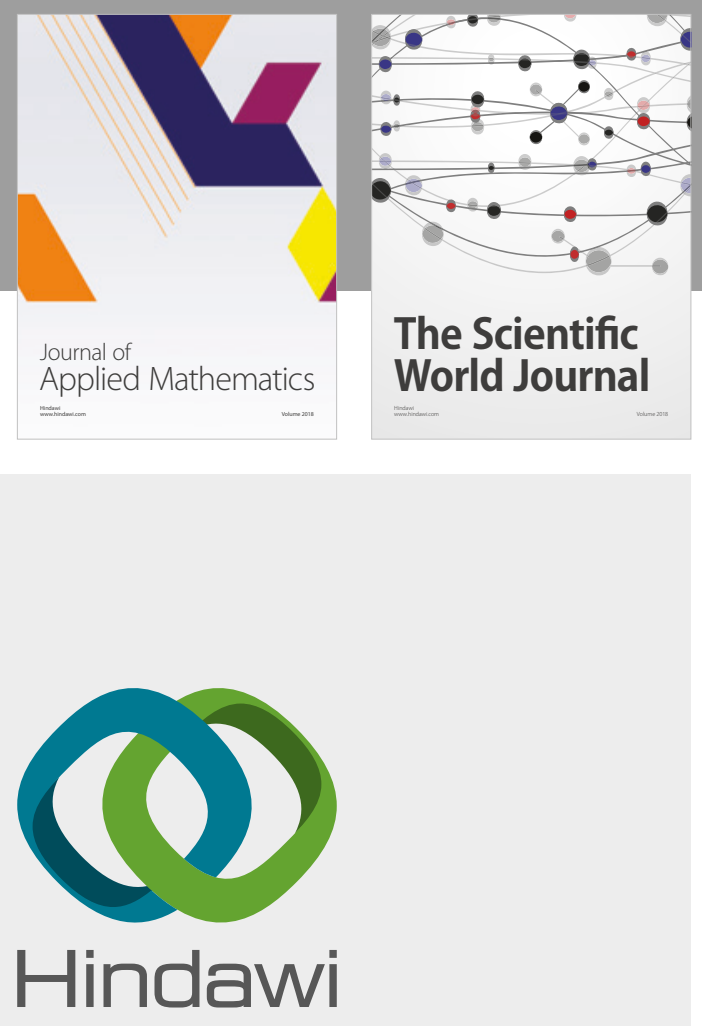

Submit your manuscripts at

www.hindawi.com

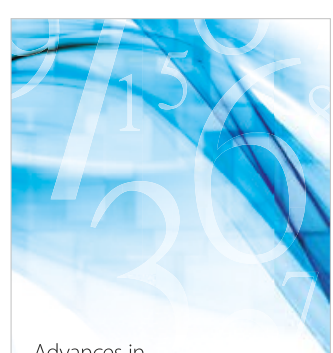

Advances in
Numerical Analysis
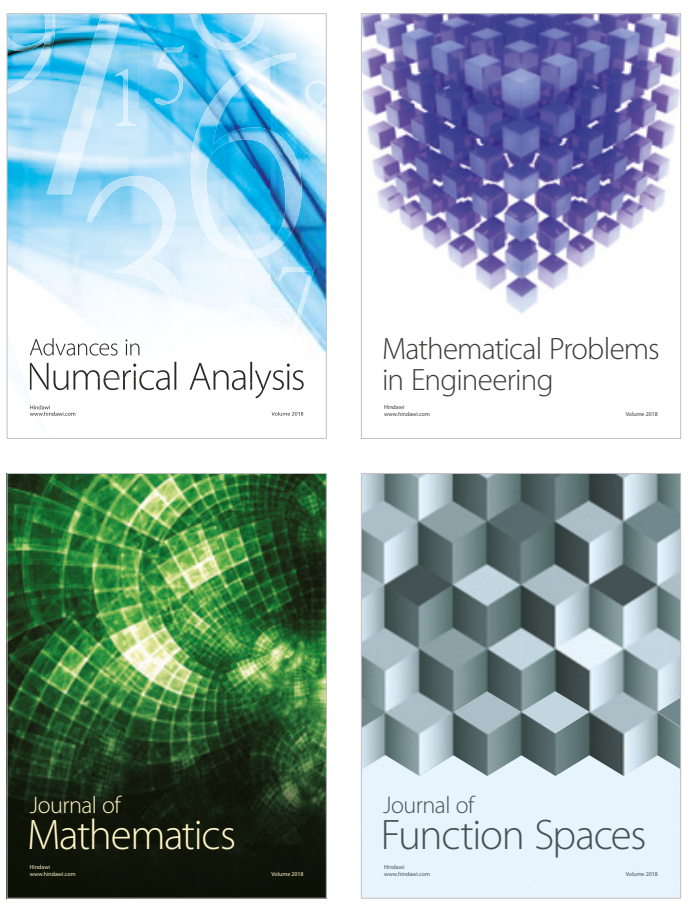

Mathematical Problems in Engineering

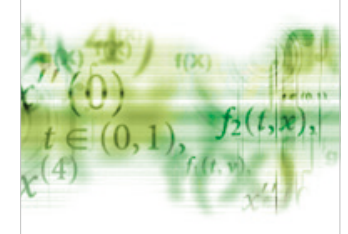

International Journal of

Differential Equations

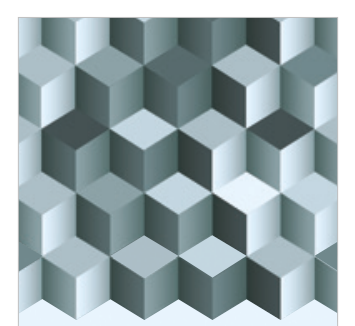

Journal of

Function Spaces

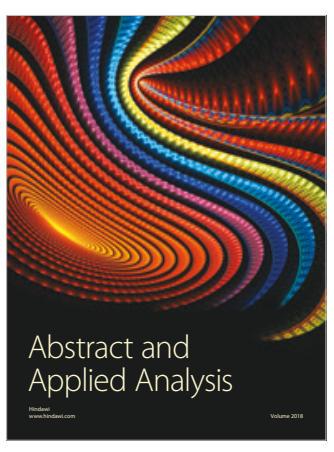

The Scientific

World Journal

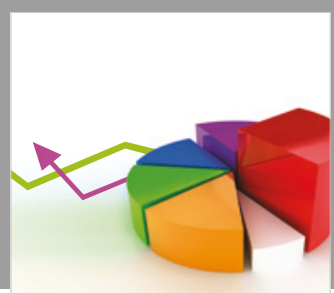

Journal of

Probability and Statistics
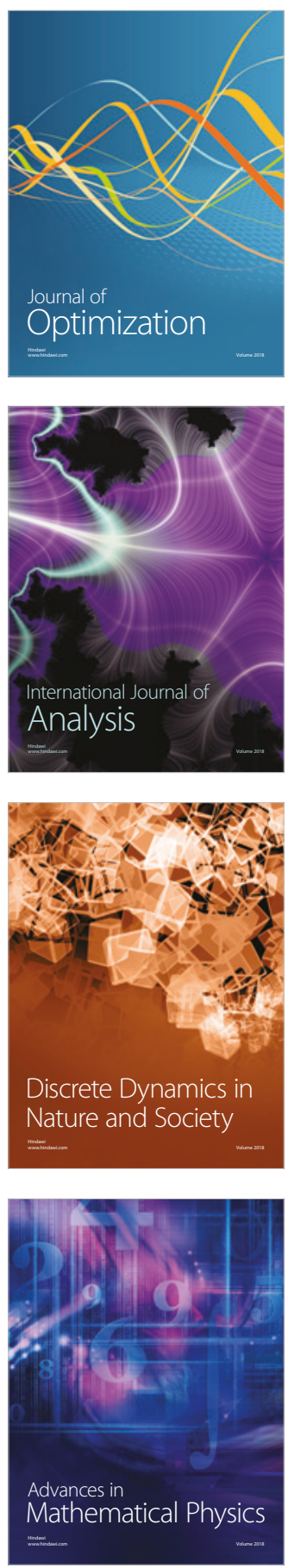